\title{
CURRICULUM VITAE - VICTOR ALGIRDAS SNIECKUS
}

Queen's University

Department of Chemistry

Tel: (613) 533-2239

Kingston, Ontario K7L 3N6

Fax: (613) 533-6089

E-mail: snieckus@chem.queensu.ca

\section{PERSONAL DATA}

Born

Citizenship

Married

Two Children

Languages

Outside Interests

\section{EDUCATION}

Preuniversity

B.Sc. (Honors)

M.Sc.

Ph.D.

Postdoctoral

\section{ACADEMIC POSITIONS}

University of Waterloo

Queen's University

Brock University

Snieckus Innovations
Kaunas, Lithuania, 1 August, 1937

Canadian

Anne Cecilia (Pinkham) Snieckus

Darius Victor (b. 1967)

Naomi Marie (b. 1970)

English, French, German, Lithuanian, Estonian

History of Science, Jazz, Table Tennis
Germany, 1945-48

Alberta, Canada, 1948-55

University of Alberta, 1959

University of California, Berkeley, 1961 (with D.S. Noyce)

University of Oregon, 1965 (with V. Boekelheide)

National Research Council, Ottawa, 1965-66 (with O.E. Edwards)

$\begin{array}{ll}\text { Assistant Professor } & 1967-71 \\ \text { Associate Professor } & 1971-79 \\ \text { Professor } & 1979-92 \\ \text { Monsanto/NSERC Ind Res Chair } & 1992-98 \\ \text { Bader Chair in Organic Chemistry } & 1998- \\ \text { Bader Chair in Organic Chemistry } & \\ \text { Emeritus } & 2009- \\ \text { Adjunct Professor, Organic Chemistry } & 2009- \\ & \\ \text { Director } & 2009-\end{array}$

\section{RESEARCH INTERESTS}

The Snieckus group has contributed to the development and application of the directed ortho metalation reaction (DoM) and used it as a conceptual platform for discovery of new efficient methods for the 
regioselective synthesis of polysubstituted aromatics and heteroaromatics. The directed remote metalation (DreM) reaction and DoM - linked transition metal catalyzed cross coupling (especially Suzuki-Miyaura) were first uncovered in his laboratories. These have found broad application especially in pharmaceutical industry (Research director quote: "When we say 'let's do it by Snieckus chemistry', we know exactly what we mean.” DoM has received NAME REACTION status (Kürti \& Czakó book).

\section{PROFESSIONAL ACTIVITIES}

Organic Editor

Volume Editor

\section{Editor}

Editor, American Continents

Editor, American Continents

Topical Editor

Advisory Editorial Board

Advisory Editorial Board

Advisory Editorial Board

Advisory Board

\section{Advisory Board}

Editorial Board Member

Editorial Board Member

Board of Consulting Editors

Editorial Committee

President/Executive Committee

Chairman, Organic Division

Councilor, Organic Division

Alternate Councilor (DOC)

Member

Member

Member, International Advisory Committee

Member, International Advisory Committee

CIC/CSC Organic Division:

NSERC Grant Selection Committee

Polanyi Prize Committee

Rutherford Medal Committee
Canadian Journal of Chemistry, 1988-2012

Science of Synthesis, Vols 8a-b (The Alkali Metals), Snieckus, V. Majewski, M. Eds. Georg Thieme Verlag, Stuttgart, 2005. A 1700-page, two-volume, 42 author, work dealing with the last decade of Alkali Metals, especially Lithium, chemistry aimed to become the successor of the eminent Houben-Weyl series as the practical, hands-on bible of synthetic methods for industial/academic chemists.

Advances in Carbanion Chemistry, Ed-in-Chief, A.

Padwa, Jai Press, 1988-1999

SYNLETT, 1990-

SYNFACTS, 2005-

Polycyclic Aromatic Compounds, 1988-

Journal of Organic Chemistry, 1984-89

Progress in Heterocyclic Chemistry, 1988-95

Chemistry \& Biodiversity, 2003-

International Symposium on Polynuclear Aromatic Hydrocarbons, 1987-93

International Society Carbanion Chemistry (ISCC), Madison, WI, 2006-

Organic Process Research \& Development, 2000-

Advanced Synthesis and Catalysis, 2000-2012

Tetrahedron/Tetrahedron Letters 2003-2007

International Society on Polynuclear Aromatic

Hydrocarbons, 1989-95.

International Society on Heterocyclic Chemistry

(ISHC), 1990-92

American Chemical Society, 1989-90

American Chemical Society, 1993-96

American Chemical Society, 1996-2002

International Activities Committee, ACS, 1994-2008

International Advisory Board: International

Symposium on Process Chemistry (ISPC 2011)

$18^{\text {th }}$ International Congress of Heterocyclic

Chemistry (ICHC), 2000, 2007

International Conference on Heteroatom Chemistry, 2002

Representative from ACS Organic Division, 1989-

Member 1996-99

1988-91 (Chair, 1991)

1997-2000 (Chair, 2000) 
Society Memberships:

Combinatorial Chemistry Research

Grants Program Committee

Member, Awards Selection Committee

Chairman, Awards Selection Committee

Chairman, Awards Selection Committee

Member, International Advisory Committee

Consultantships:

Society Memberships
American Chemical Society (ACS), Canadian Society of Chemistry (CSC), International Society for Heterocyclic Chemistry, American Association for the Advancement of Science

Member, 2001-2002

Herbert C. Brown Award for Creative Research in Synthetic Methods, 2003-2005

Katritzky Award, International Society of Heterocyclic Chemistry, 2007

THIEME/IUPAC PRIZE in Synthetic Organic Chemistry, 2000-

18th International Symposium on Carbanion Chemistry (ISCC), 2007

Albany Molecular Research (AMRI), 1999-2009 NPS (Allelix Biopharmaceuticals), 1994-2000 Sanofi-Aventis (Hoechst Marion Roussel), 19962006

Apotex Pharmachem Inc., 1997-2002

Cambrex 2000-2001

Ceregen, 1989-1995

Archimica (formerly Clariant), 2000-2008

Bristol-Myers Squibb (Dupont Merck), 1989-

BMS Canada, 2003-2007

Kyowa Hakko, 1999-2009

Merck, Sharp \& Dohme, UK, 1997-2000

Morphochem 2000-2002

OSI Pharmaceuticals (CADUS) 1996-2009

Pharmacia (Monsanto/Searle), 1989-2003

Syntex, 1987-1993

DuPont Stine, 1989-1992, 2002-

Major Laboratories, Scientific Advisory Board, 2003-

Bioblocks, Advisor Board, 2003-

ViroChem, Montreal, 2008-2009

American Chemical Society

Canadian Society of Chemistry

International Society for Heterocyclic Chemistry

Science for Peace

\section{FELLOWSHIPS AND AWARDS (listed since 1990)}

1991

1992-97

1993

1993

1993
Universitat Basel Chair, Switzerland

NSERC/Monsanto Industrial Research Chair in Chemical Synthesis and Biomolecular Design

The Alfred Bader Award in Organic Chemistry

Japan Society for the Promotion of Science (JSPS) Fellowship

Fellow of the Royal Society of Canada 
1994

1995

1996

1996

1996

1997

1999

2000

2000-2001

2001

2001

2001

2001

2001

2001

2002

2003

2003

2005

2005

2005

2006

2006

2007

2007

2007

2008

2008

2009

2009

2009

2009

2010

2011

2011

2013

Gastprofessor, University of Zurich, Switzerland (June 6-July 15, 1994)

Gastprofessor, University of Innsbruck, Austria (May 1-June 15, 1995)

Glaxo Wellcome Professorship, Duke University (January 24-February 26, 1996)

Humboldt Research Award (Humboldt-Forschungspreis)

University of Oregon Alumni Achievement Award in Chemistry

R.U. Lemieux Award for Organic Chemistry

Fellow, Lithuanian Academy of Sciences

100 Milestones of $20^{\text {th }}$ Century Canadian Chemistry Award

Killam Research Fellowship

ACS Arthur C. Cope Scholar Award

International Society for Heterocyclic Chemistry Award

Japan Society for the Promotion of Science, Invitational Fellowship for Research

AstraZeneca Excellence Award in Organic Synthesis

Boehringer-Ingelheim Distinguished Lecturer

Royal Society of Chemistry, Perkin Division, Lecturer

Gedimino Order of the Republic of Lithuania

H.C. Brown Lecturer

Craig Visiting Professor, Australian National University, Canberra

Bernard Belleau Award of the Canadian Society for Chemistry

Novartis Chemistry Lectureship

Aventis Vision Lecturer

AstraZeneca Lecturership

GlaxoSmithKline, Grey College Fellowship, University of Durham, UK

Bristol-Myers-Squibb Lecturer, North Jersey ACS Section

Fellow, International Society of Heterocyclic Chemistry (ICHC)

Aldrich Lectureship, Scripps Research Institute, La Jolla

Boehringer-Ingelheim Lecturer, UQAM, Montreal, April, 2008.

Givaudan/Karrer Visiting Professor, University of Zurich, Organic Chemistry Institute

ICHC Symposium Dedicated to V. Snieckus, St. John's, Newfoundland, August, 2009

Novartis Lecturer, Central European Countries, Prague, Budapest, Bratislava, April $18^{\text {th }}-24^{\text {th }}, 2009$

Fellow, American Chemical Society ( 1 of 2 non-Americans of 150 recipients)

Honous causa, Technical University of Tallinn, Estonia

Lithuanian Academy of Sciences Laureate

Excellence In Research, Queen's University

Alumni Award for a Distinguished BSc Graduate, University of Alberta

Global Lithuanian Award from the President of the Republic of Lithuania,

Dalia Grybauskaite

\section{PLENARY AND SPECIAL INVITED LECTURESHIPS (249 total, listed since 1993)}

1993 - ACS Spring Symposium: Organic Synthesis and Methodology, Rochester, N.Y., March 15-16, 1993.

- $\quad$ Gordon Research Conference, New Hampton, N.H., July 5-9, 1993.

- $\quad$ 34th IUPAC Congress, Beijing, China, August 15-20, 1993.

- $\quad$ 11th Symposium on Chemistry of Heterocyclic Compounds, Prague, Czechoslovakia, August 29-September 4, 1993.

- 1993 CHEMETALL Hydrid Symposium, Goslar, Germany, September 29-October 1, 1993.

- $\quad$ VII Mokslo Ir Kurybos Simpoziumas, Chicago, IL, November 24-28, 1993. 
19th IUPAC Symposium on the Chemistry of Natural Products, Karachi, Pakistan, January 16-20, 1994.

- $\quad$ 4th French/American Chemical Society, New Orleans, January 30-February 3, 1994.

- 29th ESF/EUCHEM Conference on Stereochemistry-Bürgenstock, Switzerland, May 1-7, 1994.

- $\quad$ Lilly Research Laboratories Lecturer at Ohio State University, Columbus, Ohio, September 22, 1994.

- 1994-1995-Organic Synthesis Seminar Series, The DuPont Experimental Station, Wilmington, DE, October 31, 1994.

- $\quad$ 17th Annual Meeting of the Brazilian Chemical Society, Caxambu, Brazil, May 24-27, 1994.

- $\quad$ International Isotope Society Meeting, Pearl River, November 4, 1994.

1995 - Canadian Workshop in Organic Synthesis, CSC Conference, Guelph, Ontario, May 28June 1, 1995.

- Pre-Congress Symposium "Modern Aspects of Organic Synthesis", in honour to Prof. Sauter, June 12, 1995.

- $\quad$ 5th Blue Danube Symposium on Heterocyclic Chemistry, Casta-Papiernicka, June 14-17, 1995.

- $\quad$ 15th International Congress of Heterocyclic Chemistry, Taipei, Taiwan, R.O.C., "Anionic Friedel - Crafts Equivalents. Regiospecific Construction of Thioxanthones, Xanthones, and Dibenzo[b,e]Phosphorinones", August 6-11, 1995.

- 6th Québec/Ontario Symposium in Synthetic and Bioorgainc Chemistry, McGill University, Montréal, PQ, "Metalation: The Point of Departure from New Synthetic Aromatic/Heteroaromatic Chemistry", October 28, 1995.

- Invited lecturer, Organic/Inorganic Seminar Series, "The Directed ortho Metalation Reaction: A New Chapter for Streitwieser and Heathcock?", University of Chicago, December 8, 1995.

- $\quad$ Laval University, Quebec City, PQ"Les réactions et stratégies de métallation en synthèse organique", January 18, 1996.

- $\quad$ University of Oregon, "Sightseeing the Metallated Flatlands", on the occasion of receiving the University of Oregon Alumni Achievement Award in Chemistry, March 8, 1996.

- $\quad$ 211th ACS, New Orleans, Louisiana, March 24-28, 1996.

- $\quad$ 79th Canadian Society for Chemistry Conference and Exhibition, Memorial University of Newfoundland, "Sparteine-Mediated Enantioselective Carbanionic Processes. Direct Methods for the Synthesis of Chiral Alkylbenzenes and Ferrocenes with Planar Chirality" June 23-26, 1996.

- $\quad$ 15th National Organic Conference, Central Queensland University, Australia, "New Regio- and Enantio-Selective Carbanion Mediated Processes of Synthetic Utility" June 30July 5, 1996.

- XVIIth International Conference Organometallic Chemistry, Brisbane, Australia, 7-12 July, 1996.

- $\quad$ 212th ACS, Orlando, Florida, August 25-30, 1996.

1997 - “Overview: Catalysis in Asymmetric Synthesis”, Winter Conference on Medicinal and Bioorganic Chemistry, Steamboat Springs, Colorado, January 26-31, 1997.

- $\quad$ 117th Annual Meeting of the Pharmaceutical Society of Japan, March 26-28, 1997.

- Symposium on Drug Discovery in Neuroscience and Pain, Astra Research Centre, Montreal, May 15-16, 1997. 
- $\quad$ "Synthetic Observations from the Flatlands. Contributions Towards Chemical Synthesis of Aromatics". 80th CSC, Windsor, Ontario, June 1-4, 1997.

- US-Baltics Workshop in Environmental Chemistry, ACS Conference, Palanga, Lithuania, June 11-14, 1997.

- $15^{\text {th }}$ International Symposium on Synthesis in Organic Chemistry, St. Catherine's College, Oxford, UK, July 21-24, 1997.

- Gordon Research Conference on Organic Reactions and Process, Henniker, NH, August, 1997.

- $\quad$ Syntex Lecturer, Colorado State University, Fort Collins, Colorado, October 19-21, 1997.

- J. Cartier Colloque, Lyon, France, December 7-11, 1997.

- $\quad$ UCI Synthesis Symposium, Irvine, California, December 13, 1997.

1998 - ICOS-12, Venice, Italy, June 28-July 2, 1998.

- $\quad$ The10th Sendai International Scientific Symposium, Sendai, Japan, July 5, 1998.

- $\quad$ The $9^{\text {th }}$ International Symposium on Novel Aromatic Compounds, Hong Kong, August 27, 1998.

- $\quad$ The Impact of Organic Synthesis on Drug Discovery, Heidelberg Symposium, August 30, 1998.

- $\quad$ Merck Frosst Lecturer, University of Montreal, November 18, 1998.

1999 - The Third Lake Tahoe Symposium on Molecular Diversity, California, January 24-29, 1999.

- $\quad 12^{\text {th }}$ Annual Organic Chemistry Day, University of Missouri, Columbia, Missouri, May 1, 1999.

- $\quad$ Combinatorial Chemistry Symposium, $82^{\text {nd }}$ CSC, Toronto, ON, June 1-2, 1999.

- $\quad$ Heterocycles Symposium, 82 ${ }^{\text {nd }}$ CSC, Toronto, ON, June 1-2, 1999.

- $\quad$ Gordon Research Conference on Organic Reactions and Processes, Henniker, NH, July 11$16,1999$.

- $\quad$ IUPAC conferences on Organometallic Chemistry, Versailles, France, July 18-22, 1999.

- $\quad 5^{\text {th }}$ Annual Organic Colloquium, Proctor \& Gamble, Cincinnati, OH, July 30, 1999.

- $\quad 17^{\text {th }}$ International Congress of Heterocyclic Chemistry, Vienna, August 1-6, 1999.

- $\quad$ KOPO Symposium, Ulm, Germany, September 22, 1999.

- Metal Catalyzed Cross Coupling Reactions, Society Chemical Industry, UK, October 5, 1999.

- $\quad 7^{\text {th }}$ Wyeth-Ayerst Research Award Lecture, Wyeth Ayerst and Columbia University, December 16-17, 1999.

2000 - INFORMEX, New Orleans, Louisiana, March 15-18, 2000.

- $\quad 1^{\text {st }}$ Florida Heterocyclic Conference, Gainesville, Florida, March 8-10, 2000.

- $\quad$ Organic Process Research and Development (OPRD) Conference, July, 2000.

- Gordon Research Conference on Natural Products Chemistry, Henniker, NH, July 30, 2000.

- $\quad$ Groupe d'Etudes de Chimie Organique (GECO) XXXXI, Nièvre, France, August 27September 1, 2000.

- $\quad$ Gulf Coast Conference, Pensicola, Florida, September 14-16, 2000.

- $\quad$ Royal Society of Chemistry Heterocyclic Group Meeting, London, UK, September 26, 2000 .

$2001 \quad$ - $\quad 4^{\text {th }}$ Winter Conference on Medicinal and Bioorganic Chemistry (WCMBC), Steamboat Springs, Colorado, January 28-February 2, 2001.

- $\quad$ Sixth International Conference on Heteroatom Chemistry, Kodz, Poland, June 22-27. 
- $\quad$ 8th International Society of Heterocyclic Chemistry, Yokohama, Japan, July 29-Aug 3, 2001.

- $\quad$ Post-OMCOS-XI Conference, Kyoto, Japan, July 27-29, 2001.

- $\quad$ Cope Award Symposium, 222nd ACS Meeting, Chicago, August 2001.

- $\quad$ Cornforth - Eaborn Lecture, University of Sussex, UK, August 31, 2001.

- $\quad$ Second European Catalysis Symposium, Italy, September 23-26, 2001.

- $\quad$ IBC (Molecular Diversity) Meeting, San Diego CA, October 10-12, 2001.

- $\quad$ Dupont Pharma Symposium, University of California, San Diego CA, December 10, 2001.

- $\quad$ Royal Society of Chemistry, Newcastle-upon-Tyne, 2001.

- $\quad$ Second European Catalysis Symposium, Italy, September 23-26, 2001.

- $\quad$ IBC (Molecular Diversity) Meeting, San Diego CA, October 10-12, 2001.

- $\quad$ Dupont Pharma Symposium, University of California, San Diego CA, December 10, 2001.

- $\quad$ Royal Society of Chemistry, Perkin Division, Glasgow, December, 2001.

- $\quad$ Millennium Pharmaceuticals, Boston, December, 2001.

- $\quad$ ICNPA Conference, Gottingen, Germany, May 9-12, 2002.

- $\quad$ SECO Conference, St. Jean de Monts, France, May, 2002.

- $\quad$ F/5 Conference, Ulm, Germany, May, 2002.

- $\quad$ Morphochem, Germany, May, 2002.

- $\quad$ ICOS-14, University of Auckland, New Zealand, July 12, 2002.

- $\quad$ Merck Research Laboratories, September 6, 2002.

- $\quad$ Distinguished Merck Lecturer, Merck, University of Peurto Rico, September 30, 2002.

- Ontario/Quebec Mini-Symposium on Synthetic and Bioorganic Chemistry, Canada, October 8-10, 2002.

- Queen's Award Celebration, Symposium, Scientific Update, London, UK, October 30, 2002.

- $\quad$ Syngenta, Jealotts Hill, UK, November 20, 2002.

$5^{\text {th }}$ Winter Conference on Medicinal and Bioorganic Chemistry (WCMBC), Steamboat Springs, Colorado, January 27-31, 2003.

- $\quad$ Kyowa Hakko, Mishima Research Laboratories, Kyowa Hakko, Japan, July 3, 2003.

- H.C. Brown $20^{\text {th }}$ Anniversary Lecture, West Lafayette, Indiana, September 23, 2003.

- $\quad$ Albany Molecular Research Center, Albany, New York, September 10, 2003.

- $\quad$ Arfvedson-Schlenk Symposium, GDCh Jahrestagung, Munich, Germany, October 7, 2003.

- $\quad$ Serono Reproductive Biology Institute, Boston, Massachusettes, November 5, 2003.

- Distinguished Lecturer, DuPont Crop Protection Center, Stine, November 7, 2003.

2004 - Krohn Symposium, Universität Paderborn, Paderborn, Germany, April 23, 2004.

- $\quad$ Canada-France Congress, Nice, France, April 26, 2004.

- $\quad 50^{\text {th }}$ Anniversary of A.N. Nesmeyanov Institute of Organoelement Compounds, Moscow, Russia, June, 2, 2004.

- $\quad$ Queguiner Symposium, Rouen, France, June 24, 2004.

- $\quad 7$ th International Symposium on Carbanion Chemistry (ISCC-7), Alicante, Spain, July 710, 2004.

- $\quad$ ICOS-15, Nagoya, Japan, August 1-6, 2004.

2005 - $\quad 6^{\text {th }}$ Winter Conference on Medicinal and Bioorganic Chemistry (WCMBC), Steamboat Springs, Colorado, January 23-29, 2005.

- $\quad$ Current Aspects of Synthetic Organic Chemistry Symposium (Symposium in Honor of 
Alfred Bader), 229 ${ }^{\text {th }}$ American Chemical Society National Meeting, March 13-March16, 2005.

- $\quad$ Vision Symposium, Aventis, Bridgewater, New Jersey, May 24, 2005.

- $\quad 88^{\text {th }}$ Canadian Society for Chemistry Conference, Saskatoon, Saskatchewan, May 27-June $1,2005$.

- $\quad$ Lithuanian Academy of Arts \& Sciences Meeting, Vilnius, Lithuana, June 30-July 3, 2005.

- $\quad 14^{\text {th }}$ European Symposium on Organic Chemistry, Helsinki, Finland, July 4-July 7, 2005.

- $\quad$ Polish Aacemy of Sciences Meeting (Symposium in Honor of M. Makosza), July 14-16, 2005.

- $\quad$ AstraZeneca Canada Lecturer, 2005.

- $\quad$ Novartis Distinguished Lectureship, 2005.

- $\quad$ Pacifichem 2005, Honolulu, Hawaii, December 15-20, 2005.

2006 - Pfizer Distinguished Lectureship, York University, Toronto, ON, April 20, 2006.

- $\quad$ Novartis Distinguished Lectureship, Cambridge, Massachusetts, May 2, 2006.

- $\quad$ Merck Frosst Distinguished Lectureship, University of Victoria, Victoria, B.C., May 4, 2006.

- ICOS-16, Mérida, Yucatán, México, June 11-15, 2006.

- 2006 GRC on Heterocyclic Compounds, Salve Regina College, New Port, RI, July 2-7, 2006.

- $\quad 2006$ GRC on Natural Products, Tilton, NH, July 23-28, 2006.

- $\quad$ CRC International Symposium, Purdue University, West Lafayette, IN, September 15-16, 2006.

- $\quad$ Frontiers in Organic Chemistry Symposium, University of Illinois, Urbana, IL, October 14, 2006.

2007 - Synthetic Heterocyclic Chemistry Conference, Barcelona, Spain, April 12-13, 2007.

- $\quad$ High Throughput Medicinal Chemistry III, RSC Symposium, Harlow, UK, May 1, 2007.

- $\quad$ New Jersey ASC Spring Symposium, Newark, NJ, May 24, 2007.

- $\quad$ Organic Chemistry: Perspectives on the $21^{\text {st }}$ Century IV, Barton Confernce, St. Lucia, May 27-June 2, 2007.

- 21 st International Symposium for Polycyclic Aromatic Compounds, Trondheim, Norway, August 5-10, 2007.

2008 - Z Zing Conference on Natural Products, Antigua, January, 2008.

- $\quad$ 6th Rencontres de Chimie Organique (RCO), Gif-sur-Yvette, France, April 9, 2008.

- $\quad$ Donald S.Matteson Honorary Symposium, Pullman, WA, August, 2008.

- $\quad$ Albert I. Meyers In Memoriam Symposium, Fort Collins, CL, September, 2008.

- $\quad$ Glaxo-SmithKline Symposium, September, 2008.

- Forum on Asymmetric Synthesis and Technology (FAST), Princeton, NJ, October 27-29, 2008.

2009 - Organic Winter Meeting, Norwegian Organic Chemical Society Meeting, January 8, 2009.

- $\quad$ Zing Conference on Catalytic Reactions, Antigua, March, 2009.

- $\quad 22^{\text {nd }}$ Conference on Advances in Organic Synthesis, Krapacz, Poland, July, 2009.

- $\quad 22$ nd International Congress on Heterocyclic Chemistry, St. John's, Newfoundland and Labrador, August 2-7, 2009.

2010 - Synthesis and Applications of Boron Compounds, $93^{\text {rd }}$ Canadian Society for Chemistry Conference, May 29-June 2, 2010. 
- $\quad$ 6th BASF Boron Conference, Ludwigshafen, Germany, June 14-16, 2010.

- $\quad$ Gordon Research Conference, Organic Reaction and Processes, Smithfield, RI, July 1823, 2010 .

- $\quad$ ICOS -18, Bergen, Norway, August 1-6, 2010.

$2011 \quad 12^{\text {th }}$ Florida Heterocyclic and Synthetic Conference, Gainsville, Florida, March, 2011.

- H.C. Brown Symposium honouring Professor Akira Suzuki, 241 ACS Meeting, Anaheim, CA: March, 2011.

- $\quad$ First Mexican Meeting On Pure And Applied Chemistry, $43^{\text {rd }}$ IUPAC, Mexico City, Mexico, May 16-20, 2011.

- $\quad$ Modern Synthetic Methods \& Chiral Europe Conference, Edinburgh, Scotland, May 2011.

- $\quad$ Heck Symposium, University of Delaware, Newark, Delaware, May, 2011.

- $\quad 1^{\text {st }}$ Pharmaron Symposium on Synthetic and Medicinal Chemistry, Pharmaron Beijing Co., Ltd, Beijing, China, September 17, 2011.

- $\quad$ YoungChem Conference, Krakow, Poland, October 12-16, 2011.

2012 - 2012 Excellence in Research Award Lecture, Queen's University, Kingston, March 20, 2012.

- $\quad$ Manfred Hesse Symposium, Zurich, Switzerland, March 22, 2012.

- $\quad$ Victor Grignard Centenary Symposium, Lyon, France, June 22-23, 2012.

2013 - A Symposium in Honour of David N. Harpp, 96th Canadian Chemistry Conference and Exhibition in Québec, Quebec, May 26-30, 2013.

- $\quad$ Grasmere Heterocyclic Chemisty conference, Grasmere, UK, May 9-13 ${ }^{\text {th }}, 2013$.

- $\quad$ GECO (Groupe d'Etude de Chimie Organique), Batz-sur-mer, France, August 25-30, 2013.

- International Symposium on Carbanion Chemistry (ISCC-10), Kyoto, Japan, September 23-26, 2013.

\section{SHORT COURSES (92 TOTAL, LISTED SINCE 1993)}

The Directed ortho Metalation (DoM) Reaction in Organic Synthesis and Methods and Strategies in Heteroaromatic Metalation, Merck Frosst, Montreal, Quebec, January 12 and 13, 1993.

The Directed ortho Metalation (DoM) Reaction in Organic Synthesis and Methods and Strategies in Heteroaromatic Metalation, Rhone-Poulenc Rorer, Collegeville, PA, March 22-24, 1993.

Methods and Strategies in Heteroaromatic Metalation, Anaquest Inc., Murray Hill, N.J., May 10, 1993.

The Directed ortho Metalation (DoM) Reaction in Organic Synthesis and Methods and Strategies in Heteroaromatic Metalation, EGIS Pharmaceuticals Ltd., Budapest, Hungary, September 6-8, 1993.

The Directed ortho Metalation (DoM) Reaction in Organic Synthesis and Methods and Strategies in Heteroaromatic Metalation, presented in collaboration with Scientific Update, France, October 4-8, 1993. 
The Directed ortho Metalation (DoM) Reaction in Organic Synthesis and Methods and Strategies in Heteroaromatic Metalation, Monsanto Company, St. Louis, Missouri, November 16, 18, 19, 1993.

The Directed ortho Metalation (DoM) Reaction in Organic Synthesis, Instituto de Quimica, Unicamp, Campinas, Brazil, May, 1994.

The Directed ortho Metalation (DoM) Reaction in Organic Synthesis, Gesellschaft Deutscher Chemiker (GDCH), Frankfurt, Germany, October 11-13, 1994.

The Directed ortho Metalation (DoM) Reaction in Organic Synthesis and "Methods and Strategies in Heteroaromatic Metalation, Dow Elanco, Indianapolis, Indiana, November 28-December 2, 1994.

"The Directed ortho Metalation (DoM) Reaction in Organic Synthesis and "Methods and Strategies in Heteroaromatic Metalation", Novo Nordisk, Denmark, Sept. 20-22, 1995.

The Directed ortho Metalation (DoM) Reaction in Organic Synthesis and "Methods of Aromatic Synthesis", DoM and Cross Coupling Reactions", Zeneca Pharmaceuticals, U.K., Oct./Nov., 1995.

"The Directed ortho Metalation (DoM) Reaction in Organic Synthesis" and "Aryl-Aryl Cross Coupling Methods and Strategies in Synthesis", Merck Sharp \& Dohme, Essex, U.K., Nov. 7-8, 1995.

The Directed ortho Metalation (DoM) Reaction in Organic Synthesis and "Methods and Strategies in Heteroaromatic Metalation. Synthetic Aspects of the Formation of the Aryl-Aryl Bond. The Heck Reaction.", Zeneca Pharmaceuticals, U.K., Sept. 2-4, 1996.

The Directed ortho Metalation (DoM) Reaction in Organic Synthesis and "Methods and Strategies in Heteroaromatic Metalation. Synthetic Aspects of the Formation of the Aryl-Aryl Bond. The Heck Reaction.", Rhone Poulenc Rorer, U.K., Sept. 5-6, 1996.

The Directed ortho Metalation (D0M) Reaction in Organic Synthesis and "Transition Metal Catalyzed Processes and Strategies in Aromatic and Heteroaromatic Synthesis". CIBA, Switzerland, Sept. 10, 1996.

Methods of Aromatic Synthesis, DoM and Cross Coupling Reactions, Zeneca Pharmaceuticals, Cheshire, England, Oct. 28-30, 1996. Deployment, Kodak, Rochester, New York, U.S.A., April 2-4, 1997. 
Scientific Update, East Sussex, U.K., April 28-30, 1997.

The Aryl-Aryl Cross Coupling Reaction. Methods, Strategies, and Synthetic Deployment, SmithKine Beecham Pharmaceuticals, Essex, U.K., July 17-18, 1997.

The DoM/HetDoM - Aryl-Aryl Cross Coupling Nexus. Methods, Strategies and Synthetic Deployment, Brantford Chemicals, August 1, 1997.

The DoM Reaction: New Strategies and Methodologies in Aromatic and Heteroaromatic Syntheisis, University of Darmstadt, Germany, Sept 22-24, 1997.

The DoM Reaction in Organic Synthisis: Methods and Strageies in Heteroaromatic Directed Metalation, Astra Arcus, Rochester, New York, U.S.A., March 9-11, 1998.

The Aryl-Aryl Cross Coupling Reaction. Methods, Strategies, and Synthetic Deployment, Rohm and Haas, Pensylvania, U.S.A., March 18-20, 1998.

The DoM Reaction in Organic Synthesis: Methods and Strategies in Heteroaromatic Directed Metalation, Biochem Pharma, Montreal, October 21, 1998.

1999 The Aryl-Aryl Cross Coupling Reaction. Methods, Strategies, and Synthetic Deployment, Scientific Update, UK, March 29-30, 1999.

Cross Coupling Strategies and Methods in Aromatic and Heteroatoromatic Synthesis, Scientific Update, UK, October 6, 1999.

The DoM Reaction in Organic Synthesis" and " The Aryl-Aryl Cross Coupling Reaction. Methods, Strategies, and Synthetic Deployment", Boehringer-Ingelheim, January 17, 2000.

Modern Aspects in Synthetic Heterocyclic Chemistry, Scientific Update, UK, June, 2000 .

Cross Coupling Strategies and Methods in Aromatic and Heteroaromatic Synthesis, Scientific Update, Stratford-upon-Avon, UK, June 27-29, 2001.

Directed ortho Metalation - Transition Metal Catalyzed Combinations in Heteoraromatic Synthesis, Morphochem, Munchen, Germany, July 2-4, 2001.

The Heteroaromatic Directed ortho Metalation (HetDoM) Reaction, ACS Perspectives, Barcelona, Spain, February $24^{\text {th }}, 2002$.

Catalytic Cross Coupling Chemistry of Aromatic Molecules, Kodak, Rochester, New York, May 6-8, 2003.

Catalytic Cross Coupling Reactions in Aromatic and Heteroaromatic Synthesis. Mechanism, Method, and Strategy. Pfizer, Groton, Connecticuit, October 26-27, 2003.

Cross Coupling Reactions in Aromatic and Heteroaromatic Synthesis, Scientific Update, Cambridge, UK, December 10-11, 2003. 
Modern Methodologies in Aromatic and Heteroaromatic Anionic and Transition Metal Catalyzed Chemistry, Albany Molecular Research, Inc., September 29-30, 2004.

Cross Coupling Reactions in Aromatic and Heteroaromatic Synthesis, Scientific Update, Surrey, UK, October 7-8, 2004.

Cross Coupling Reactions in Aromatic and Heteroaromatic Synthesis, Scientific Update, Surrey, UK, October 5-7, 2005.

Cross Coupling Reactions in Aromatic and Heteroaromatic Synthesis, Serono, Boston, November 10-11, 2005.

Cross Coupling Reactions in Aromatic and Heteroaromatic Synthesis, ScheringPlough Research Institute, Boston, MA, April 27, 2006.

Cross Coupling Reactions in Aromatic and Heteroaromatic Synthesis, Xenon-Pharma, Vancouver, B.C., May 5, 2006.

Cross Coupling Reactions in Aromatic and Heteroaromatic Synthesis, Scientific Update, Surrey, San Francisco, CA, May 8-10, 2006.

Cross Coupling Reactions in Aromatic and Heteroaromatic Synthesis, Scientific Update, Surrey, Nice, France, October 3-5, 2006.

Cross Coupling Reactions in Aromatic and Heteroaromatic, Sanofi-Aventis, Newark, New Jersey, November 30-December 1, 2006.

Metal-catalyzed Annulation to Aromatics and Heteroaromatics. Emerging Strategies for Heterocycylic Construction, AMRI, Albany, NY, March 28, 2007.

The Directed ortho Metalation (DoM) Strategy for Synthetic Aromatic and Heteroaromatic Chemistry, Tallinn University of Technology, Tallinn, Estonia, September 17-18, 2007.

The Directed ortho Metalation (DoM) Strategy for Synthetic Aromatic and Heteroaromatic Chemistry, Vilnius University, Vilnius, Lithuania, September 20-21, 2007.

Catalytic Cross Coupling Reactions in Aromatic \& Heteroaromatic Synthesis, Scientific Update, Barcelona, Spain. October 9-11, 2007.

Catalytic Cross Coupling Reactions in Aromatic \& Heteroaromatic Synthesis, OSI, Farmingdale, New Jersey, December 20-21, 2007.

Catalytic Cross Coupling Reactions in Aromatic \& Heteroaromatic Synthesis, Merck \& Co., Rahway, NJ, January 14-15, 2008.

Catalytic Cross Coupling Reactions in Aromatic \& Heteroaromatic Synthesis, Merck \& Co., Westpoint, PA, January 17-18, 2008.

Connecting ortho Metlation to Transition Metal Catalyzed Reactions in Aromatic and Heteroaromatic Synthesis, Singapore, February 18-22, 2008. 
Organic Synthesis Begins with Lithium, Florence, Italy, April 1-3, 2008.

Cross Coupling Strategies and Methods in Aromatic \& Heteroaromatic Synthesis, Budapest, Hungary, April 15-18, 2008.

Cross Coupling Strategies and Methods in Aromatic \& Heteroaromatic Synthesis, Dublin, Ireland, October 7-9, 2008.

Catalytic Cross Coupling Reactions in Aromatic \& Heteroaromatic Synthesis, Gilead Sciences, Inc., Foster City, California, December 11-12, 2008.

2009

Organic Synthesis Begins with Lithium (with M. Majewski), Solvay Pharmaceuticals, Weesp, The Netherlands, January 20-21, 2009.

Organic Synthesis Begins with Lithium (with M. Majewski), Solvay Pharmaceuticals, Hannover, Daix, Germany, May 11-15, 2009.

Catalytic Cross Coupling Reactions in Aromatic \& Heteroaromatic Synthesis, Genentech, South San Francisco, CA, May 2010.

2011

Directed ortho Metalation, Transition Metal Catalyzed Coupling, and C-H Activation. Contemporary Synthetic Strategies for Aromatics and Heteroaromatics, Abbott, Cambridge, MA, May 2011.

Catalytic Cross Coupling Reactions In Aromatic \& Heteroaromatic Synthesis, Sunovion, Marlborough, MA, November 9-11, 2011.

Catalytic Cross Coupling Reactions In Aromatic \& Heteroaromatic Synthesis, Universidad del Pais Vasco, Bilbao, Spain, May 9-10, 2012.

Directed ortho Metalation (DoM): Contemporary Synthetic Strategies for Aromatics and Heteroaromatics, SciUP, Edinburgh, Scotland - September 17th-19th 2012.

Catalytic Cross Coupling Reactions in Aromatic \& Heteroaromatic Synthesis, Scientific Update, Prague, Czech Republic, November 2012.

\section{INVITED SEMINARS (listed since 1993)}

University of Georgia, February 24, 1993.

Clemson University, South Carolina, February 25, 1993.

FMC Corporation, Gastonia, North Carolina, February 26, 1993.

M.I.T., Cambridge, MA, April 8, 1993.

Rutgers University, Newark, N.J., May 11, 1993.

Schering-Plough Research Institute, June 8, 1993.

Allelix Biopharmaceuticals Inc., Mississauga, Ontario, July 29, 1993.

Roche Research Centre, Hertfordshire, U.K., October 8, 1993.

Acadia University, Wolfville, N.S., October 25, 26, 1993.

Syntex Research, Palo Alto, CA, December 6, 1993.

Genentech Inc., South San Francisco, CA, December 7, 1993.

Corvas Internation, San Diego, CA, December 8, 1993. 
Department of Sciene and Technology, Calcuttta, India, January 21, 1994.

Calcutta University, Calcuttta, India, January 21, 1994.

Jadavpur University, Calcutta, India, January 22, 1994.

Indian Association for the Cultivation of Science, Jadavpur, Calcutta, India, January 24, 1994.

Wayne State University, March 3, 1993.

Warner-Lambert Co., Ann Arbor, MI, March 4, 1994.

Marion Merrell Dow Co., Cincinnati, Ohio, March 10, 1994.

Burroughs Wellcome, Research Triangle Park, N.C., March 11, 1994.

University of Connecticut, Storrs, CT, March 30, 1994.

Hoffmann-La Roche, Basel, Switzerland, April 29, 1994.

Universidade Federal do Rio de Janeiro, Brazil, May, 1994.

Universidade Federal de Minas Gerais, Brazil, May, 1994.

Ciba-Geigy, Basel, Switzerland, June 28, 1994.

ETH Zentrum, Switzerland, June 30, 1994.

Lilly Corporate Center, Indianapolis, Indiana, September 20, 1994.

University of Pisa, Pisa, Italy, October 5 and 6, 1994.

Neurosearch, Copenhagen, Denmark, October 7, 1994.

Hoechst AG, Frankfurt, Germany, October 10, 1994.

Johnson Matthey, Philadelphia, October 25, 1994.

University of Alabama, Tuscaloosa, November 10, 1994.

University of Alabama, Birmingham, November 11, 1994.

Boehringer Ingelheim, Ridgefield, Connecticut, November 14, 1994.

University of Western Ontario, November 16, 1994.

ACIC Canada, Brantford, Ontario, January 26, 1995.

Pfizer Inc, Groton, CT, February 15, 1995.

Bristol-Myers, Wallingford, February 16, 1995.

Laboratoires Fournier S.C.A., Dijon, France, March 20, 1995.

Iowa State University, Ames, Iowa, April 14, 1995.

Bristol Meyers, Princeton, N.J., April 19, 1995.

Bristol Meyers, New Brunswick, N.J., April 20, 1995.

Boehringer Mannheim GmbH, Mannheim, Germany, 1995.

University of Twente, Enschede, The Netherlands, 1995.

Ciba-Geigy Limited, Basle, Switzerland, June 22, 1995.

Eastman Kodak Company, July 13, 1995.

BioChem Therapeutic Inc., Montréal, PQ, October 27, 1995.

Rhone Poulenc Rorer, Terlings Park, U.K., November 6, 1995.

University of Chicago, Chicago, IL December 8, 1995.

Université Laval, Québec City, PQ, January 18, 1996.

Sintese de Cumestanos e Tiocumestanos, Universidade Federal do Rio de Janeiro, May 27-30, 1996.

Reilly Industries, Indianapolis, IN, September 12, 1996.

Kansas State University, Lawrence, KS September 24, 1996.

Agouron Pharmaceuticals, San Diego, CA, October 15, 1996.

Corvas International, San Diego, CA, October 16, 1996.

Athena Neurosciences, Inc., South San Francisco, October 17, 1996.

Argonaut Technologies, Inc., San Carlos, CA, October 21, 1996.

Roche Bioscience, Palo Alto, CA, October 22, 1996.

Amgem, Thousand Oaks, CA, October 23, 1996.

Zeneca Huddersfiled Works, Huddersfield, England, October 31, 1996.

Sepracor, Inc., Marlborough, MA, November 6, 1996.

McGill University, Montréal, PQ, January 21, 1997.

Novo Nordisk, Mont Tremblant, PQ, February 4, 1997. 
Astra Draco, Malmo, Sweden, February 4, 1997.

Astra Arcus, Stockholm, Sweden, February 11, 1997.

Astra Hassle, Gothnberg, Sweden, February 12, 1997.

Astra Charnwood, Leics, U.K., February 13, 1997.

Bristol-Myers Squibb, Montreal, PQ, March 3, 1997.

Cadus Pharmaceutical Corporation, Tarrytown, NY, March 13, 1997.

Sakai Research Labs, Osaka, Japan, March 24, 1997.

Kyowa Hakko Kogyo Co. Ltd., Tokyo, Japan, March 25, 1997.

Pfizer Limited, Sandwich, Kent, UK, May 1, 1997.

Astra Research Centre, Montreal, PQ, July 29, 1997.

Gordon Research Conference on Organic Reactions and Process, Henniker, NH, August, 1997.

Pharmacia Upjohn, Kalmazoo, Michigan, September 4-5, 1997.

Agouron, San Diego, California, October 15, 1997.

Colorado State University, Fort Collins, Colorado, October 19-21, 1997.

Terrapin Technologies Inc. San Fransisco, November 6, 1997.

BASF, Germany, December 5, 1997.

J. Cartier Colloque, Lyon, France, December 7-11, 1997.

Irvine, California, December 13, 1997.

Simon Fraser University, Burnaby, B.C., January 21, 1998.

St. Mary's University, Halifax, January 23, 1998.

Boehringer Ingelheim Pharma KG, Germany, June 23-24, 1998.

Bayer, West Haven, Conneticut, September 14, 1998.

Ontogen Corporation, California, October 19, 1998.

Viropharma, Philadelphia, November 23, 1998.

Albermarle, Louisiana, November 2, 1998.

Cadus, Tarrytown, NY, January 21, 1999.

BioChem Pharma, Quebec, February 9, 1999.

CLARIANT, Frankfurt, July 27, 1999.

Laval University, Quebec, September 6, 1999.

University of Manchester, UK, September 29, 1999.

Merck Sharpe \& Dohme, UK, September 30, 1999.

Acadia University, Nova Scotia, November 4, 1999.

Dalhousie University, Nova Scotia, November 5, 1999.

Memorial University of Newfoundland, Dec 4, 1999.

Wyeth-Ayerst, Columbia University, New York, NY, December 17, 1999.

Emisphere Technologies Inc., Tarrytown, NY, February 18, 2000.

Kyowa Hakko, Japan, April 9, 2000.

Abbott Laboratories, Chicago, Illinois, April 18, 2000.

University of Illinois, Chicago, Illinois, April 19, 2000.

Alanex, San Diego, California, April 21, 2000.

Eli Lilly, UK, November 25-27, 2000.

Imperial College, UK, November 28, 2000.

Centre National de la Recherche Scientifique, France, November 29-December 1, 2000.

AstraZeneca, Montreal, December 7, 2000.

University of Pennsylvania at Philadelphia, April 16, 2001.

Juniata College, Huntingdon PA, April 17, 2001.

Boehringer-Ingelheim, New Orleans LA, May, 2001.

Dupont Merck, Wilmington DE, May 16, 2001.

University of Girona, Italy, May 24, 2001.

Canadian Chemical Society, Montreal, June 2001.

Post-OMCOS-XI, Kyoto, Japan, July, 2001. 
Second European Catalysis Symposium, Pisa, September 2001.

Royal Society of Chemistry, Newcastle-upon-Tyne, September, 2001.

Boehringer-Ingelheim, University of New Orleans, November 16, 2001.

AstraZeneca, Delaware, November 6, 2001

$2^{\text {nd }}$ UCSD/DuPont Pharma Symposium on Organic Chemistry, December 10, 2001

Millennium Pharmaceuticals, Boston, December 14, 2001.

Royal Society of Chemistry: Perkin Division, Glasgow, December 19, 2001

Ibn Sina Conference, Egypt, February, 2001

Bristol-Myers Squibb: MIT, Cambridge, April, 2002.

Joint Chemistry/Physics Speaker Series, Trent University, Peterborough, April 3, 2002

International Conference on Natural Products and Analogues, Gottingen, May, 2002

Semaine d'Etudes de Chimie Organique, St. Jean de Monts, May, 2002.

CSC National Conference, Vancouver, May 2002

Fifth International Symposium on Functional Electron Systems, Ulm, Germany, June, 2002.

$9^{\text {th }}$ Blue Danube Symposium on Heterocyclic Chemistry, Tatranski Lomnica, June 2002.

Roche Symposium, University of Colorado, Colorado, June $7^{\text {th }}, 2002$

Balticum Organicum Syntheticum, Vilnius, Lithuania, June 23-27, 2002.

ICOS 14, New Zealand, July, 2002.

Oppolozer Symposium, Geneva, Switzerland, October $4^{\text {th }}-6^{\text {th }}, 2002$.

Scientific Update, New Orleans, Louisiana, November 12-15 $5^{\text {th }}, 2002$.

Walden Symposium, Riga, Latvia, October $13^{\text {th }}-14^{\text {th }}, 2003$.

Roche Carolina, Inc., Florence, South Carolina, October $22^{\text {nd }}-23^{\text {rd }}, 2003$.

CV Therapeutics, Inc., San Francisco, California, November 3-5, 2003.

Lecturer, DuPont Crop Protection Center, Stine, November 7, 2003.

Serono Reproductive Biology Institute, Boston, Massachusetts, November 5, 2003.

DuPont Stine Crop Protection, Newark, Delaware, November 6-7, 2003.

Scios, Inc., Fremont, California, February 17, 2004.

Dartmouth College, Hanover, New Hampshire, February 19, 2004.

Balticum Organicum Syntheticum, June 27-July 1, 2004.

Biogen, Cambridge, MA, September 8, 2004.

Bristol-Myers Squibb, Candiac, Quebec, September 13, 2004.

Bristol-Myers Squibb, Syracuse, New York, September 15, 2004.

BASF, Germany, October 11-13, 2004.

NERM Symposium, November 1-2, 2004.

Lund University, Sweden, December 6, 2004.

University of Stockholm, Stockholm, Sweden, December 9-10, 2004.

Bristol-Myers Squibb, Candiac, Quebec, April 19, 2005.

DuPont Stine Crop Protection, Newark, Delaware, May 16-17, 2005.

Sorono International S.A., Geneva, Switzerland, July 7, 2005.

Bristol-Myers Squibb, Candiac, Quebec, September 16, 2005.

Bristol-Myers Squibb, Wallingford, Connecticut, September 26, 2005.

Bristol-Myers Squibb, Princeton, New Jersey, September 27, 2005.

Albany Molecular Research, Inc., Syracuse, New York, September 28, 2005.

Wilfried Laurier University, Waterloo, Ontario, January 20, 2006.

Xenon-Pharma, Vancouver, B.C., May 5, 2006.

Georgia State University, Athens, Georgia, October 14, 2006.

F-Tech, Tokyo, Japan, October 26, 2006.

University of Saskatchewan, Saskatoon, SK, February 20, 2007.

University of Wisconsin, Madison, Wisconsin, April 17, 2007.

University of Connecticut, Storrs, Connecticut, April 25, 2007.

Bristol-Myers Squibb, New Brunswick, New Jersey, April 26, 2007 
University College Dublin, Dublin, Ireland, May 3, 2007.

Aldrich Lectureship, Scripps Research Institute, La Jolla, CA, May 11, 2007.

AMRI, Albany, NY, July 10, 2007.

AMRI, Syracuse, NY, July 11, 2007.

Vertex, Boston, MA, July 12, 2007.

University of Strathcylde, Glasgow, Scotland, September 26, 2007.

Heterocyclic and Synthesis Group Meeting, Organon, Scotland, September 27-29, 2007.

Boehringer Ingelheim, Biberach, Germany, October 12, 2007.

AMRI, Albany, NY, November 16, 2007.

Sanofi-Aventis, Bridgewater, NJ, December 7, 2007.

Kyowa-Hakko, Sakai, Japan, December 10 - 14, 2007.

OSI Pharmaceuticals, December 20-21, 2007.

Antigua Natural Products Conference, January 10-13, 2008.

Bristol-Myer Squibb, New Brunswick, NJ, February 11, 2008.

Bristol-Myers Squibb, Lawrenceville, NJ, February 12, 2008.

Bristol-Myers Squibb, Wakefield, Connecticut, February 13, 2008.

AMRI Budapest, Budapest, Hungary, April 14, 2008.

Sanofi-Aventis, Budapest, Hungary, April 15-16, 2008.

University of Quebec in Montreal, April 28, 2008.

Brock University, September 12, 2008.

ACS Prospectives, Boston, MA, September 29, 2008.

Almac Sciences, Dublin, Ireland, October 10, 2008.

GlaxoSmithKline, Raleigh, North Carolina, October 13, 2008.

Albany Molecular Research Inc., Syracuse, New York, December 7, 2008.

Kyowa Hakko Kirin Co. Ltd., Sakai, Japan, December 15, 2008.

Kyoto University, December 17, 2008.

Hoffmann-La Roche, Nutley, NJ, March 26, 2009.

Wyeth Pharmaceuticals, Pearl River, NJ, May 28, 2009.

BMS, Wallingford, June, 2009.

Amgen, Thousand Oaks, California, October 26, 2009.

Bernd Giese Symposium, Basel, Switzerland, June 4, 2010.

$93^{\text {rd }}$ CSC Meeting, Toronto, Ontario, May 30-June 3, 2010.

Brock University, Synthetic Organic Chemistry in Canada: A 50-Year Selective Perspective, April 17,

2010.

PACIFICHEM Conference: Honolulu, Hawaii, December 15-20, 2010.

Pfizer, Groton, Connecticut, April 4 ${ }^{\text {th }}, 2011$.

Amgen, Cambridge, MA, June 20, 2011.

Arfvedson-Schlenk Award Symposium, Bremen, Germany, September 7, 2011.

Shanghai Institute of Organic Chemistry, Shanghai, China, September 14, 2011.

Peking University, Beijing, China, September 16, 2011.

University of Konstanz, Kontansz, Germany, October 10, 2011.

University of Alberta, Edmonton, Alberta, December 5, 2011.

Universidad del Pais Vasco, Bilbao, Spain, May 8, 2012.

Oxford University, England, May 22, 2012.

Uppsala University, Uppsala, Sweden, May 25, 2012.

BMS, Wallingford, June 10, 2012.

Boehringer-Ingelheim, Ridgefield, CT, February 11, 2013.

University of Oregon, Eugene, Oregon, June 7, 2013. 


\section{TEACHING ACTIVITIES}

\section{University of Waterloo}

Courses taught range from 1st year General Chemistry to graduate courses. Experience in teaching general chemistry, all levels of undergraduate organic chemistry, special topics in organic chemistry (heterocycles, physical organic, synthesis, natural products, biosynthesis, bioorganic chemistry, environmental chemistry). Enrollments ranged from 20-75 students.

In the 1975-95 period, taught, every year, Chemistry in Modern Society, a course designed for "poets" but usually taken by a broad mix and level of undergraduate students varying from arts to engineering, from 1st year to 4th year. Attendance of 150-300 range. Topics covered include Chemical Carcinogens, Chemical Warfare Agents, Drugs in Sports, Chemical Messages Make Scents, DNA Profiling, Aspects of Stratospheric Chemistry, some of which are given by guest lecturers.

From beginning of academic career, taught, on a regular basis, graduate courses in organic chemistry including Spectroscopic Techniques, Heterocyclic Chemistry, Free Radical Chemistry, Biogenesis and Biosynthesis, Natural Products, Synthetic Methods, and Strategies in Synthesis.

\section{Queen's University}

Continuing teaching activities, $1^{\text {st }}$ year organic chemistry, upper year organic synthesis and heterocyclic chemistry, and graduate synthetic, heterocyclic, and biosynthesis since 1998. Organization of special course in Medicinal and Pharmaceutical Chemistry of invited lecturers from the pharma and agrochemical industries to present topics of current research in discovery programs of drug and agrochemical interest.

\section{CONFERENCE PROCEEDINGS (1 ${ }^{\text {st }}: 1969$. Listed since 1993)}

1. Directed ortho-Metalation Mediated $\mathrm{E}^{+}$-Introduction: Regiospecific Synthesis of Fluorinated Aromatics. F. Beaulieu, V. Snieckus, W. Han, C.K. Murphy, F.A. Davis 205th ACS National Meeting, Denver, CO, March 28-April 2, 1993. Abstr. 172. (Oral)

2. $\mathrm{Ni}(\mathrm{O})$-Catalyzed Cross Coupling of Aryl S-Thiocarbamates and Aryl Triflates with Grignard and Zinc Reagents Respectively: Directed ortho-Metalation-Cross Coupling Connections. F. Beaulieu, C. Quesnelle, V. Snieckus 205th ACS National Meeting, Denver, CO, March 28-April 2, 1993. Abstr. 173.

3. Aromatic Metallation Aromatique. Methodology and Synthetic Consequences Synthetiques. ALFRED BADER AWARD LECTURE. V. Snieckus 76th CIC Conference, Sherbrooke, Quebec, May 30-June 3, 1993. Abstr. OR-B1 177.

4. Metalation of N,N Diethyl-O-5-Carbomyloxyindole. E.J. Griffen, V. Snieckus 76th CIC Conference, Sherbrooke, Quebec, May 30-June 3, 1993. Abstr. OR-C7 371.

5. Intramolecular Aryl to a-Amidoyl Radical 1,5-Hydrogen Atom Transfer Reactions. Highly Diastereoselective Route to Substituted $\alpha$-Alanine Derivatives. F. Beaulieu, V. Snieckus 76th CIC Conference, Sherbrooke, Quebec, May 30-June 3, 1993. Abstr. OR-E6 569.

6. Preparation of Macrocyclic Host Molecules for the Analysis and Complexation of Polychlorinated Dibenzo-p-Dioxins. M. Campbell, J.M. Wiebe, V. Snieckus 76th CIC Conference, Sherbrooke, Quebec, May 30-June 3, 1993. Abstr. OR-F8P 686.

7. $\mathrm{Ni}(\mathrm{O})$-Catalyzed Cross Coupling of Aryl Triflates with Organozinc Reagents. C. Quesnelle, V. Snieckus 76th CIC Conference, Sherbrooke, Quebec, May 30-June 3, 1993. Abstr. OR-G6 819.

8. Combined Metalation - Cross Coupling Approach to Phenanthroviridin and Related Natural Products. B.-p. Zhao, V. Snieckus 76th CIC Conference, Sherbrooke, Quebec, May 30-June 3, 1993. Abstr. OR-G6 818. 
9. Directed ortho Metalation Mediated $\mathrm{F}^{+}$-Introduction. Regiospecific Synthesis of Fluorinated Aromatics. F. Beaulieu, V. Snieckus 76th CIC Conference, Sherbrooke, Quebec, May 30-June 3, 1993. Abstr. OR-B8P 192.

10. Preparation of Macrocyclic Host Molecules for the Complexation of a-Amino Acids Using Lithiation and Cross Coupling Strategies. M. Campbell, V. Snieckus 76th CIC Conference, Sherbrooke, Quebec, May 30-June 3, 1993. Abstr. OR-F8P 685.

11. Heterocyclic Excursions Based on the Directed Metalation-Cross Coupling Symbiosis. V. Snieckus Gordon Research Conference, New Hampton, NH, July 5-9, 1993.

12. Combined Metalation - Cross Coupling Approach to Phenanthroviridin and Related Natural Products. B.-p. Zhao, V. Snieckus 14th International Conference on Heterocyclic Chemistry (ICHC), Antwerp, Belgium, August, 1-6, 1993 Abstr. PO2-84.

13. Intramolecular Aryl to $\alpha$-Amidoyl Radical 1,5-Hydrogen Atom Transfer Reactions. Highly Diastereoselective Route to Substituted $\beta$-Alanine Derivatives. F. Beaulieu, V. Snieckus 14th International Conference on Heterocyclic Chemistry (ICHC), Antwerp, Belgium, August, 1-6, 1993. Abstr. OP-Sy-8.

14. Directed ortho Metalation-Cross Coupling Connections. Ni(O)-Catalyzed Cross Coupling of Aryl S-Thiocarbamates and Aryl Triflates with Grignard and Organo Zinc Reagents. F. Beaulieu, C. Quesnelle, V. Snieckus 14th International Conference on Heterocyclic Chemistry (ICHC), Antwerp, Belgium, August, 1-6, 1993. Abstr. PO2-85.

15. New Synthesis of Condensed Anthracene and Phenanthrene PAHS Via Aromatic Metalation Cross Coupling Tactics. B.-p. Zhao, S. Chowdhury, V. Snieckus 14th International Symposium on Polycyclic Aromatic Compounds, Osage Beach, Missouri, September 8-11, 1993. Abstr. OC5.

16. Combined Metalation - Cross Coupling Approach to Phenanthroviridin and Related Natural Products. V. Snieckus 34th IUPAC Congress, Beijing, China, August 15-20, 1993 Abstr. I-1006.

17. Remote Directed Metalation of Diaryl Sulfone $o$-Amides and $o$-Carbamates. Synthesis of Diaryl Sulfones and New Route to Substituted Thioxanthen-9-One 10,10-Dioxides. F. Beaulieu and V. Snieckus 206th ACS Meeting, Chicago, IL, August 22-27, 1993. Abstr. ORGN 81.

18. Expedient Synthesis of 3,3'-Disubstituted 1,1'-BI-(2-Phenols) (Bipols) by Directed ortho Metalation. A.S. Parsons, J.M. Garcia and V. Snieckus 206th ACS Meeting, Chicago, IL, August 22-27, 1993, Abstr. ORGN 422.

19. Intramolecular Aryl to $\alpha$-Amidoyl Radical 1,5-Hydrogen Atom Transfer Reactions. Highly Diastereoselective Route to Substituted $\beta$-Alanine Derivatives. F. Beaulieu and V. Snieckus 206th ACS Meeting, Chicago, IL, August 22-27, 1993, Abstr. ORGN 421.

20. Metal(1)ation: The Point of Departure for New Synthetic Aromatic Chemistry. V. Snieckus 1993 CHEMETALL Hydrid Symposium, Goslar, Germany, September 20-October 1, 1993.

21. [1,5]-H Atom Translocations in Thiobenzamides. J. Arora and V. Snieckus Guelph-Waterloo Centre for Graduate Work in Chemistry $\left(\mathrm{GWC}^{2}\right)$ Saturday Seminar, Waterloo, November 13, 1993.

22. Combined Metalation-Cross Coupling Approach to Phenanthroviridin Aglycon and Related Natural Products. Z. Baoping and V. Snieckus Guelph-Waterloo Centre for Graduate Work in Chemistry $\left(\mathrm{GWC}^{2}\right)$ Saturday Seminar, Waterloo, November 13, 1993.

23. Preparation of Macrocyclic Host Molecules for the Analysis and Complexation of Polychlorinated Dibenzo-p-Dioxins. M. Campbell and V. Snieckus Guelph-Waterloo Centre for Graduate Work in Chemistry $\left(\mathrm{GWC}^{2}\right)$ Saturday Seminar, Waterloo, November 13, 1993.

24. Total Synthesis of Biruloquinone. B. Chapell and V. Snieckus Guelph-Waterloo Centre for Graduate Work in Chemistry $\left(\mathrm{GWC}^{2}\right)$ Saturday Seminar, Waterloo, November 13, 1993.

25. Synthesis of New Dibenzopyranones as Ellagic Acid Antimutagenic Congeners. A. Coelho and V. Snieckus Guelph-Waterloo Centre for Graduate Work in Chemistry (GWC ${ }^{2}$ ) Saturday Seminar, Waterloo, November 13, 1993.

26. 1,4-rearrangements of Benzylic Carbamates. I. Ionica and V. Snieckus Guelph-Waterloo Centre 
for Graduate Work in Chemistry (GWC ${ }^{2}$ ) Saturday Seminar, Waterloo, November 13, 1993.

27. Studies Toward the Total Synthesis of Defucogilvocarin M. C. James and V. Snieckus GuelphWaterloo Centre for Graduate Work in Chemistry (GWC $\left.{ }^{2}\right)$ Saturday Seminar, Waterloo, November 13, 1993.

28. A Few New Areas of Vinyl Carbamates. B. Joseph and V. Snieckus Guelph-Waterloo Centre for Graduate Work in Chemistry $\left(\mathrm{GWC}^{2}\right)$ Saturday Seminar, Waterloo, November 13, 1993.

29. $\mathrm{Ni}(\mathrm{O})$-Catalyzed Cross Coupling of Aryl Triflates with Organozinc Reagents. C. Quesnelle and V. Snieckus Guelph-Waterloo Centre for Graduate Work in Chemistry $\left(\mathrm{GWC}^{2}\right)$ Saturday Seminar, Waterloo, November 13, 1993.

30. Stereoselective Synthesis of Dihydroxyethylene Dipeptide Isostere. M. Tsukasaki and V. Snieckus Guelph-Waterloo Centre for Graduate Work in Chemistry $\left(\mathrm{GWC}^{2}\right)$ Saturday Seminar, Waterloo, November 13, 1993.

31. The Emerging Centrality of the Directed ortho Metalation Reaction in Synthetic Aromatic Chemistry. V. Snieckus VII Mokslo ir Kurybos Simpoziumas, Chicago, IL, November 24-28, 1993; Festkolloquium, Moderne Aspekte der Organischen Synthese, Wien, June 12, 1995.

32. Synthesis of 3,3'-Disubstituted 1,1'-Bis(2-Phenols (Bipols) by Directed ortho Metalation and Cross Coupling. A.S. Parsons, J. Garcia, and V. Snieckus 32nd Convention of the South African Chemical Institute South Africa, January 30-February 3, 1994 (Oral).

33. $\alpha$-Lithiated Vinyl Carbamates as Acetaldehyde Dianion Equivalents. Synthesis of Dihydroxyethylene Dipeptide Isostere. M. Tsukazaki and V. Snieckus 207th ACS Meeting, San Diego, CA, March 13-18, 1994. Abstr. ORGN 161.

34. Combined Metalation and Cross-Coupling Approaches to Biruloquinone and Defucogilvocarin M. B.A. Chapell, C.A. James, and V. Snieckus 207th ACS Meeting, San Diego, CA, March 13-18, 1994. Abstr. ORGN 162.

35. [1,5-Hydrogen Atom Translocation in o-Halothiobenzamides. J. Arora and V. Snieckus 77th CIC Conference, Winnipeg, Manibota, May 29-June 2, 1994. Abstr. OR-C6, 254.

36. 1,4-Wittig Rearrangement of Benzylic Carbamates. M. Leite, B. Chapell, I. Ionica, V. Snieckus 77th CIC Conference, Winnipeg, Manibota, May 29-June 2, 1994. Abstr. OR-C6, 255.

37. Ni(O)-Cataylzed Cross Coupling of Aryl O-Carbamates and Aryl Triflates with Grignard Reagents. M.N. Leite, S. Sengupta, D.S. Raslan, C. Quesnelle, and V. Snieckus 17th Annual Meeting of the Brazilian Chemical Society, Campinas, Brazil, May 24-27, 1994. Abstr. QO-65

38. Studies in the Synthesis of Radermachol. M.A.F. Brandao, A.B. de Oliveira, D.S. Raslan, and V. Snieckus. 17th Annual Meeting of the Brazilian Chemical Society, Campinas, Brazil, May 24-27, 1994. Abstr. QO-66.

39. Anionic $\mathrm{O} \rightarrow \mathrm{C}$ Carbamoyl Migration of 2-Methyl Aryl O-Carbamates. Regiospecific Route to Substituted Benzofuranones. M.A.J. Miah, M. Tsukazaki, M. Wicki, T. Nguyen, M. Kerr, and V. Snieckus. 209th National ACS Meeting, Anaheim, CA, April 2-6, 1995. Abstr. ORGN 374.

40. Anionic Friedel-Crafts Equivalents. Regiospecific Construction of Thioxanthones, Xanthones, and Dibenzo[b,e] Phosphorinones. F. Beaulieu, P. Moreau, O.B. Familoni, I. Ionica, M. Gray, V. Snieckus 209th National ACS Meeting, Anaheim, CA, April 2-6, 1995. Abstr. ORGN 375.

41. Biaryl O-Carbamate Remote Metalation Reactions. Studies Towards the Total Synthesis of the Defucogilvocarcin Family of Antibiotics. C. James and V. Snieckus. 209th National ACS Meeting, Anaheim, CA, April 2-6, 1995. Abstr. ORGN 376.

42. $\alpha$-Aryl Vinyl Carbamates. Tandem $\beta$-Carbolithiation $\alpha$-Alkylation and [1,2]-Wittig Rearrangement.S. Superchi, B. Joseph, N. Sotomayor, Y. Jia, and V. Snieckus. 1995 CSC Conference, University of Guelph, Guelph, Ontario, May 28-June 1, 1995.

43. Substituant Correlation and Kinetic Studies of the Suzuki Cross Coupling Reaction. M. Campbell and V. Snieckus. 1995 CSC Conference, University of Guelph, Guelph, Ontario, May 28-June 1, 1995.

44. Total Synthesis of Biruloquinone. B. Chapell and V. Snieckus. 1995 CSC Conference, University 
of Guelph, Guelph, Ontario, May 28-June 1, 1995.

45. Studies Towards the Total Synthesis of the Gilvocarcins. C. James and V. Snieckus 1995 CSC Conference, University of Guelph, Guelph, Ontario, May 28-June 1, 1995.

46. Anionic $\mathrm{O} \rightarrow \mathrm{C}$ Carbamoyl Migration of 2-Methyl Aryl O-Carbamates. Regiospecific Route to Substituted Benzofuranones. M.A.J. Miah, M. Tsukazaki, M. Wicki, T. Nguyen, M. Kerr, and V. Snieckus. 15th ICHC, Taipei, Taiwan, August, 6-11, 1995.

47. Anionic Friedel-Crafts Equivalents. Regiospecific Construction of Thioxanthones, Xanthones, and Dibenzo[b,e] Phosphorinones. F. Beaulieu, P. Moreau, O.B. Familoni, I. Ionica, M. Gray, V. Snieckus. 15th ICHC, Taipei, Taiwan, August, 6-11, 1995.

48. Preparation and Complexation Studies of Macrocyclic Hosts for the Complexation of Aromatic Pollutants, poster session. V. Snieckus, M. Campbell 15th ICHC, Taipei, Taiwan, August, 6-11, 1995.

49. New Synthetic Methods for Heterocycles Based on Carbanion-Mediated Processes. W.I. Iwema Bakker, A. da Silva, I. Ionica, M. Gray, O.B. Familoni, F. Beaulieu, J.A. Glaenzer, C. James and

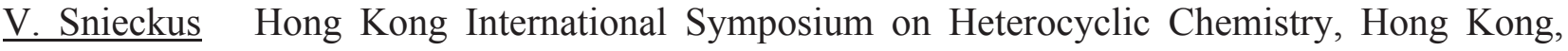
August 13-16, 1995.

50. Anionic $\mathrm{O} \rightarrow \mathrm{C}$ Carbamoyl Migration of 2-Methyl Aryl O-Carbamates. Regiospecific Route to Substituted Benzofuranones. M.A.J. Miah, M. Tsukazaki, M. Wicki, T. Nguyen, M. Kerr, and V. Snieckus. Hong Kong International Symposium on Heterocyclic Chemistry, August 13-16, 1995.

51. Benzeneoid Functionalization of Indoles and Tryptophols via Combined Directed Ortho Metalation-Cross Coupling Methodology. E. Griffen, D. Roe, N. Somera and V. Snieckus 210th ACS National Meeting, Chicago, Illinois, U.S.A., August 20, 1995.

52. Sparteine-Mediated Enantioselective Lateral Metalation of Ortho-Ethyl Aryl O-Carbamate. A. Roglans, M. Tinkl and V. Snieckus 210th ACS National Meeting, Chicago, Illinois, U.S.A., August 20, 1995.

53. Transition Metal Catalyzed Aryl-Aryl Cross Coupling Reactions. Comparative Synthetic and Mechanistic Studies. M. Campbell, C. Quesnell, V. Snieckus 1995 Pacifichem Conference, Honolulu, Hawaii, December 17-22, 1995.

54. Sparteine-Mediated Enantioselective Lateral Metalation of ortho-Ethyl Aryl $O$-Carbamate. M. Tinkl, A. Roglans, T. Kimachi, and V. Snieckus 211th American Chemical Society Meeting, New Orleans, March 24-29, 1996.

55. A Direct and Highly Enantioselective Synthesis of Ferrocenes with Planar Chirality by (-)Sparteine-Mediated Lithiation. M. Tsukazaki, M. Tinkl, A. Roglans, B.J. Chapell, N.J. Taylor and V. Snieckus 211th American Chemical Society Meeting, New Orleans, March 24-29, 1996.

56. Anionic Friedel-Crafts Equivalents. Regiospecific Construction of Dibenzo[b,e]-Phosphorinones, Xanthones, and Acridones. Poster. M. Gray, O.B. Familoni, I. Ionica, J.A. Glänzer, S. Macneil, L. Briggs and V. Snieckus 211th American Chemical Society Meeting, New Orleans, March 2429, 1996.

57. Sintese de Cumestanos e Tiocumestanos A.L. Coelho, V. Snieckus. Sociedade Brasileira de Quimica, 19.a Reuniao Anual, Poços de Caldas, 27-30 May, 1996.

58. Sintese Total Da 3,4,8,9,10-Pentaidroxidibenzo[b,d]Piranona-6 Uma Substancia Natural Das Flores De Tamarix Nilotica A.L. Coelho, V. Snieckus. Sociedade Brasileira de Quimica, 19.a Reuniao Anual, Poços de Caldas, 27-30 May, 1996.

59. Sparteine-Mediated Enantioselective Carbanionic Processes. Direct Methods for the Synthesis of Chiral Alkylbenzenes and Ferrocenes with Planar Chirality. B.J. Chapell, T. Kimachi, A. Roglans,

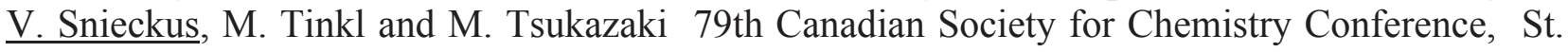
John's, Nfld., June 22-26, 1996.

60. Anionic Friedel-Crafts Equivalents: A New Regioselective Route to Polysubstitued Acridones. $\underline{\text { S. }}$ MacNeil, M. Gray, J. Glanzer, L. Briggs, and V. Snieckus. 79th Canadian Society for Chemistry Conference, St. John's, Nfld., June 22-26, 1996.

61. Anionic Friedel-Crafts Equivalents. A New Regioselective Methodology for Polysubstituted 
Xanthones. Kinsman, and V. Snieckus. 79th Canadian Society for Chemistry Conference, St. John's, Nfld., June 22-26, 1996.

62. Biaryl $O$-Carbamate Metallation Reactions. Studies in Natural Product Synthesis. C. James, and V. Snieckus. 79th Canadian Society for Chemistry Conference, St. John's, Nfld., June 22-26, 1996.

63. Probing the Directed Ortho Metalation Friedel-Crafts Complementarity. T. Kimachi, Y. Jia, L. Jin, and V. Snieckus. ECHET'96 Conference, electronic conference via Emory Univ., Atlanta, June 24-July 22, 1996.

64. (-)-Sparteine Mediated Enantioselective Carbolithiation $\alpha$-Alkylation of $\alpha$-Aryl Vinyl $O$ Carbamate. G. Miao, and V. Snieckus. ECHET'96 Conference, electronic conference via Emory Univ., Atlanta, June 24-July 22, 1996.

65. Kinetic and Thermodynamic Stability of Lithio Anions in the Synthesis of Substituted Arylsulphonamides. O.B. Familoni, M. Maillet, and V. Snieckus. ECHET'96 Conference, electronic conference via Emory Univ., Atlanta, June 24-July 22, 1996.

66. Transmetalation Reaction of Ferrocenyl Tellurides. A. Chieffi, J.V. Comasseto and V. Snieckus.ECHET'96 Conference, electronic conference via Emory Univ., Atlanta, June 24-July 22, 1996.

67. From Flatland to Prochiral Metalation. Aiming for New Synthetic Methodologies for Aromatics and Heteroaromatics. V. Snieckus. ECHET'96 Conference, electronic conference via Emory Univ., Atlanta, June 24-July 22, 1996.

68. New Regio- and Enantio-Selective Carbanion Mediated Processes of Synthetic Utility. V. Snieckus.15th National Organic Conference, Royal Australian Chemical Institute, Yeppoon, Queensland, June 30-July 5, 1996.

69. Tandem Lateral Metalation/Cyclisation of Biphenyl Imine Derivatives as a New Entry into the 9Aminophenanthrene Skeleton. Total Synthesis of Piperolactam C. S.J. Houldsworth, L. Benesch, D. Guillaneux, P. Bury and V. Snieckus. 80th Canadian Society for Chemistry Conference, Windsor, Ontario, June 1-4, 1997.

70. The Di-t-Butylphosphinyl Group as a Directed ortho Metalation Group. Synthesis of Hindered Dialklarylphosphines. B.J. Chappel, M. Gray and V. Snieckus. 80th Canadian Society for Chemistry Conference, Windsor, Ontario, June 1-4, 1997.

71. Biaryl Anionic Remote Carbamoyl Migration Reactions. The Total Synthesis of Defucogilvocarcin V.C. James and V. Snieckus. 80th Canadian Society for Chemistry Conference, Windsor, Ontario, June 1-4, 1997.

72. Regiospecific Route to Substituted $2((3 \mathrm{H})$-Benzofuranones via Anionic $\mathrm{O} \rightarrow \mathrm{C}$ Carbamoyl Rearrangement of 2-Methyl Aryl O-Carbamates. A.V. Kalinen and V. Snieckus. 80th Canadian Society for Chemistry Conference, Windsor, Ontario, June 1-4, 1997.

73. A Strategically Convrgent Synthesis of Kinafluorenone and Phenanthroviridin: Apllications of DoM/DreM and XCOUPL Tactics to Natural Produc Synthesis. M. Stefinovic, V. Snieckus.80th Canadian Society for Chemistry Conference, Windsor, Ontario, June 1-4, 1997.

74. Synthesis of Ferrocenes with Planar Chirality by (-)-sparteine-mediated Metalation: New Developments. C. Metallinos, V. Snieckus. 80th Canadian Society for Chemistry Conference, Windsor, Ontario, June 1-4, 1997.

75. Regiospecific Benzeneoid Ring Functionalisation of Indoles, Tryptophols and Tryptamines via Directed ortho Metalation and Cross Coupling Methodologies. V. Snieckus, D.G. Roe and A.C. Kinsman. 80th Canadian Society for Chemistry Conference, Windsor, Ontario, June 1-4, 1997.

76. A New Synthetic Route to Vinyl Carbamates and Their Cross Coupling Reactions with Methoxyborates.T. Moriya and V. Snieckus. 80th Canadian Society for Chemistry Conference, Windsor, Ontario, June 1-4, 1997.

77. Applications of DoM, DreM and XCOUPL Tactics to Natural Product Synthesis; The Total Synthesis of Dehydrorabelomycin. C. Kendall, I. Mohri, V. Snieckus. 80th Canadian Society for Chemistry Conference, Windsor, Ontario, June 1-4, 1997. 
78. $\quad \alpha^{\prime}, \alpha^{\prime}$-Disilylated Tertiary Amide Directed Metalation Group as Dual ortho- and $\alpha^{\prime}$-Carbanion Synthon. P.A. Patil, M. McMahon, J.-C. Cuevas and V. Snieckus. 80th Canadian Society for Chemistry Conference, Windsor, Ontario, June 1-4, 1997.

79. Synthesis of Acridone Alkaloids by an Anionic Friedel-Crafts Equivalent. S. MacNeil, M. Gray, L.E. Briggs and V. Snieckus. 80th Canadian Society for Chemistry Conference, Windsor, Ontario, June 1-4, 1997.

80. Directed Ortho Metalation in Ferrocenes. (-)-Sparteine-Mediated Enantioselective Synthesis of Planar Chiral Ferrocenes. C. Metalinos, B.J. Chapell, R.s. Laufer, C. Bessler, A. Chieffi, B. Miao, J.V. Comasseto, V. Snieckus. $215^{\text {th }}$ American Chemical Society Meeting, Dallas, March 29-April $1,1998$.

81. Directed ortho and Remote Metalation Strategies. New Approaches to Chromenes and NaturallyOccurring Coumestans. B. A. Chauder, Alexey A. Kalinin, Victor Snieckus. $81^{\text {st }}$ Canadian Society for Chemistry Conference, Whistler, British Colombia, May 31-June 4, 1998.

82. The Directed Ortho Metalation - Suzuki Cross-coupling Connection. Methological and Total Synthesis Studies. C. Kendall, R. Milburn, o. Prien, V. Snieckus. $81^{\text {st }}$ Canadian Society for Chemistry Conference, Whistler, British Colombia, May 31-June 4, 1998.

83. $12^{\text {th }}$ International Conference in Organic Synthesis, Venice Italy, June 28-July 2, 1998.

84. Directed Ortho Metalation/Cross-Coupling Connection: Synthesis of new 3,3'-substituted binols. R. Milburn, O. Prien, V. Snieckus. $216^{\text {th }}$ American Chemical Society Meeting, Boston, August 20-24, 1998.

85. Directed Metalation/Suzuki Cross-couplingconnection: Total Synthesis Studies. C. Kendall, V. Snieckus $216^{\text {th }}$ American Chemical Society Meeting, Boston, August 20-24, 1998.

86. Directed Ortho Metalation of Aryl Sulfonamides C. Lane, V. Snieckus, M.Maillet, O.B. Familoni $216^{\text {th }}$ American Chemical Society Meeting, Boston, August 20-24, 1998.

87. Synthesis of $N$-Aryl Anthranilamides via and Anionic N-Ortho Fries Rearrangement. B. Wilson, S. MacNeil, V. Snieckus Canadian Society for Chemistry National Meeting, Calgary AB, May 27-31, 2000.

88. Synthesis of New Axially Chiral 3,3'-Diaryl BINOL and BINAP Ligands. R. Quinn, R. Milburn and V. Snieckus Canadian Society for Chemistry National Meeting, Calgary AB, May 27-31, 2000.

89. Studies Towards the Total Synthesis of Dehydrorabelomycin. C. Dexter, C. Kendall and V. Snieckus Canadian Society for Chemistry National Meeting, Calgary AB, May 27-31, 2000.

90. Selective ortho vs Lateral Metalation of Secondary and Tertiary p-Tolylsulfonamides. IpsoBromo Desilylation, Suzuki Cross Coupling Reactions and Grubbs Ring Closing Metathesis.

91. S. MacNeil, C.A.L. Lane and V. Snieckus American Chemical Society National Meeting, Washington D.C., August 19-23, 2000.

92. (-)-Sparteine-mediated Enantioselective Directed Lateral Metalation Reactions. V. Derdau, J. Faessler, and V. Snieckus American Chemical Society National Meeting, Washington D.C., August 19-23, 2000.

93. Synthesis of New C2-symmetric Chiral-bridged Biferrocenees. H. Szillat and V. Snieckus. American Chemical Society National Meeting, Washington D.C., August 19-23, 2000.

94. Synthesis of New Axially chiral 3,3'-diaryl BINOL and BINAP Ligands. V. Snieckus, R. Milburn, and R. Quinn 2000 International Chemical Congress of Pacific Basin Societies, Hawaii, December 14-19, 2000.

95. Synthesis of the C/D open-ring Macrocyclic Analog of Ergot Alkaloids via a Key Intramolecular Heck Reaction. V. Snieckus, B. Chauder and A. Kalinin 2000 International Chemical Congress of Pacific Basin Societies, Hawaii, December 14-19, 2000.

96. Synthesis of New C2-symmetric Chiral-bridged Biferrocenees. V. Snieckus and H. Szillat 2000 International Chemical Congress of Pacific Basin Societies, Hawaii, December 14-19, 2000.

97. Anionic Friedel-Crafts Equivalents: Synthesis Dibenzazepinones, Dibenzoxepinones, Dibenzothiopinones, and Dibenzophosphorinones by Directed Remote and Lateral Metalation 
Reactions. V. Snieckus, S. MacNeil, W. Familoni, P. Moreau, and B. Wilson 2000 International Chemical Congress of Pacific Basin Societies, Hawaii, December 14-19, 2000.

98. Synthesis of 3,4-dihalogenated Indoles from TIPS-protected Gramine. A Retro-Mannich-directed ortho Metalation Synthetic Connection. V. Snieckus, B. Chauder, A. Larkin 2000 International Chemical Congress of Pacific Basin Societies, Hawaii, December 14-19, 2000.

99. Synthesis of Macrocyclic Analogs of Lysergic Acid. Transition Metal Catalyzed Intramolecular Heck Reaction as the Key Step. B. Chauder, Alex Kalinine, and V. Snieckus. $4^{\text {th }}$ Winter Conference on Medicinal and Bioorganic Chemistry (WCMBC), Steamboat Springs, Colorado, January 28-February 2, 2001.

100. Related Metalations of Diarylamines. Synthesis of Anthranilates, Anthranilamides, Acridones, and Dibenzazepinones. B. Wilson, S. MacNeil, and V. Snieckus. $4^{\text {th }}$ Winter Conference on Medicinal and Bioorganic Chemistry (WCMBC), Steamboat Springs, Colorado, January 28February 2, 2001.

101. Modern Synthetic Routes to PAH. Alkyl Phenanthrenes by a Directed Metalation-Cross Coupling Methodology. X. Cai, V. Snieckus. 2001 Annual Research Symposia for Canadian Network of Toxicology Centers, Ottawa, March 20-21, 2001.

102. Combined Directed ortho and Remote Metalation and Cross-Coupling Strategies in the Synthesis

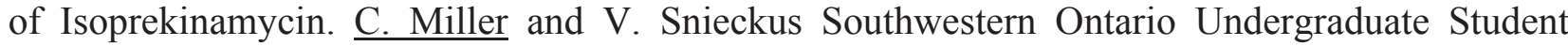
Chemistry Conference, Toronto, March 24, 2001.

103. Cai, X., Kiparissis, Y.; Blazeski, S.; Hodson, P. V.; Snieckus, V. Embryotoxicity of alkylsubstituted PAHs. CNTC (Canadian Network ofToxicology Centers) Annual Symposium, Ottawa, March 26-27, 2002.

104. Blanchet, J., Macklin, T., Snieckus, V. Directed ortho Metalation - Cross Coupling Strategies. Synthesis of Aryl Saccharins. Balticum Organicum Syntheticum, June 23-27, 2002.

105. Milburn, R., Snieckus, V. Tertiary Sulfonamide as a Latent Directed Metalation Group and Development of a New Sulfonamide N-Protecting Group. Ni-Catalyzed Reductive Cleavage and Cross Coupling Reactions of Aryl Sulfonamides with Grignard Reagents. Balticum Organicum Syntheticum, June 23-27, 2002.

106. Lee, A., Snieckus, V. Design of Teraryls with Banana Shaped Side Chains as Potential Liquid Crystal. SOUSCC, Ryerson University, April 2002.

107. Chang, M., Snieckus, V. "New Anionic Processes Using Directed orhto Metalation and Directed Remote Metalation Reactions", La Jolla, California, October, 2002. (Escorted Michelle Chang, Awardee of the Pfizer Global Research and Development Summer Undergraduate Research Fellowship).

108. M. Alessi, C. Metallinos and V. Snieckus. (-)-Sparteine-Mediated Directed ortho Metalation of Derivatives. First Case of Double Asymmetric Induction of Planar Chirality in ferrocenes and New Routes to Functionalized Planar Chiral Ferrocene Derivatives. Ontario/Quebec MiniSymposium on Synthetic and Bioorganic Chemistry, Queen's University, Kingston, October 8-10, 2002.

109. Anctil, E., Familoni, O., Snieckus, V. Synthetic Carbanionic Aromatic Chemistry. A General and Short Route to 2-Aryl-3-hydroxybenzofurans from Salicylamide Derivatives and its Application toward the Total Synthesis of Anigopressin A. Ontario/Quebec Mini-Symposium on Synthetic and Bioorganic Chemistry, Queen's University, Kingston, ON, October 8-10, 2002.

110. Zhao, Z., Snieckus, V. Ipso-Substitution of Silylated Aromatics Derived By Directed ortho Metalation. Ontario/Quebec Mini-Symposium on Synthetic and Bioorganic Chemistry, Queen's University, Kingston, ON, October 8-10, 2002.

111. McCubbin, A., Lemieux, R.P., Snieckus, V. Synthesis and Characterization of Fluorenol Liquid Crystals. Ontario/Quebec Mini-Symposium on Synthetic and Bioorganic Chemistry, Queen's University, Kingston, ON, October 8-10, 2002.

112. Laughren, J., Snieckus, V. "A New Directed Remote Metalation (DreM) Reaction of $O$-Aryl Carbamates”, Pfizer Global Research and Development Summer Undergraduate Fellowship, Ann 
Arbor, Michigan, October, 2003.

113. Alessi, M., Bieber, A., Green, L., Snieckus, V. "Progress in Directed ortho Metalation (DoM). Tetraethylphosphorodiamidate: 1. A New Directed Metalation Group. 2. In situ DoM Boronation Suzuki-Miyaura Cross Coupling of Pyridine Carboxamides", $5^{\text {th }}$ Winter Conference on Medicinal and Bioorganic Chemistry (WCMBC), Steamboat Springs, Colorado, January 27-31, 2003.

114. Alessi, M., Green, L., Snieckus, V. "One-pot DoM-Boronation and Suzuki Cross-Coupling of Pyridine Carboxamides", $39^{\text {th }}$ IUPAC Congress $/ 86^{\text {th }}$ CSC Conference, Ottawa, August $10-15$, 2003.

115. Alessi, M., Green, L., Snieckus, V. "Tetraethylphosphorodiamidate: A New Directed Metalation Group", $39^{\text {th }}$ IUPAC Congress $/ 86^{\text {th }}$ CSC Conference, Ottawa, August $10-15,2003$.

116. Anctil, E., Familoni, W., Snieckus, V. "Synthetic Carbanionic Aromatic Chemistry. A General and Short Route to 2-Aryl-3-Hydroxybenzofurans from Salicylamide Derivatives and Its Application Toward the Total Synthesis Of Anigopressin A", $39^{\text {th }}$ IUPAC Congress $/ 86^{\text {th }}$ CSC Conference, Ottawa, August 10-15, 2003.

117. Cai, X., Snieckus, V. "Synthesis of Benzo- and Indolo- [2,3-a]Carbazoles Using a Cross Coupling - Directed Remote Metalation Strategy", $39^{\text {th }}$ IUPAC Congress $/ 86^{\text {th }}$ CSC Conference, Ottawa, August 10-15, 2003.

118. Hartung, C., Nolte, B., Snieckus, V. "Towards the Synthesis of Cervinomycin. A Combined Metalation - Cross Coupling Strategy", $39^{\text {th }}$ IUPAC Congress $/ 86^{\text {th }}$ CSC Conference, Ottawa, August 10-15, 2003.

119. Jones, C., Milburn, R., Snieckus, V., Ross, G. "Directed ortho Metalation for Naphthalene 1,8Diamides. Ni(0)-Catalyzed Hydrodesulfamoylation and Cross Coupling of Arylsulfonamides", $39^{\text {th }}$ IUPAC Congress $/ 86^{\text {th }}$ CSC Conference, Ottawa, August $10-15,2003$.

120. Macklin, T., Snieckus, V. "Evaluation of the N,N-Dialkysulfamate as a New Directed ortho Metalation Group", $39^{\text {th }}$ IUPAC Congress $/ 86^{\text {th }}$ CSC Conference, Ottawa, August $10-15,2003$.

121. McCubbin, A., Tong, X., Zhao, Y., Lemieux, R., Snieckus, V. "Synthesis and Characterization of Fluorenol Liquid Crystals: Stabilization of the Smectic C* Phase Through Hydrogen Bonding", $39^{\text {th }}$ IUPAC Congress $/ 86^{\text {th }}$ CSC Conference, Ottawa, August $10-15,2003$.

122. Nolte, B., Snieckus, V. "The Directed ortho Metalation - Cross Coupling Link. Regioselective Methodologies for the Construction of Heterocycles", $39^{\text {th }}$ IUPAC Congress $/ 86^{\text {th }}$ CSC Conference, Ottawa, August 10-15, 2003.

123. Zhao, Z., Snieckus, V. "Ipso-Electrophilic Substitution of Silylated Aromatics Derived by Directed ortho Metalation Reactions", $39^{\text {th }}$ IUPAC Congress $/ 86^{\text {th }}$ CSC Conference, Ottawa, August 10-15, 2003.

124. Macklin, T., Snieckus, V. Evaluation of the $N, N$-Diethylsulfamate as a New Latent ortho Directing Metalation Group. $7^{\text {th }}$ International Symposium on Carbanion Chemistry, Alicante, Spain, July 7-11, 2004.

125. Alessi, M., Reed, M., Snieckus, V. O $\rightarrow$ C 1,5-Carbamoyl Translocation in 2-O-Carbamoyl Stilbenes. Towards the Total Synthesis of Isoprekinamycin. $7^{\text {th }}$ International Symposium on Carbanion Chemistry, Alicante, Spain, July 7-11, 2004.

126. Jones, C., Snieckus, V. Directed ortho-Metalation (DoM) of 1,8-Naphthalene Diamides. $7^{\text {th }}$ International Symposium on Carbanion Chemistry, Alicante, Spain, July 7-11, 2004.

127. Morin, J., Snieckus, V. The Directed ortho Metalation (DoM) of Naphthalene OCarbamates. Entries into Contiguously Substituted Naphthalenes, SISOIUM 2005, Montreal, Quebec, April 28-29, 2005.

128. Zhao, Y., Snieckus, V. A Short Route towards the Total Synthesis of Lysergic Acid: Application of Directed ortho Metalation (DoM) Methodology, SISOIUM 2005, Montreal, Quebec, April 2829, 2005.

129. Demchuk, O., Snieckus, V., A New Approach to the Synthesis of Scalemic Phosphorus Ligands, SISOIUM 2005, Montreal, Quebec, April 28-29, 2005. 
130. Zhao, Z., Snieckus, V. Hetero Biaryl Directed Remote Metalation (DreM). Indole N-CONEt 2 Translocation. Towards a Total Synthesis of Furostifoline, SISOIUM 2005, Montreal, Quebec, April 28-29, 2005.

131. Engqvist, R., Javaid, A., Bergman, J. Synthesis of Thienodolin, SISOIUM 2005, Montreal, Quebec, April 28-29, 2005.

132. Gan, W., Snieckus, V., Lemieux, R. Towards A New Liquid Crystal Material by A Combined Metalation-Cross Coupling Route, SISOIUM 2005, Montreal, Quebec, April 28-29, 2005.

133. Anctil, E., Familoni, W., Snieckus, V. Synthetic Carbanionic Aromatic Chemistry. A General and Short Route to 2-Aryl-3-hydroxybenzofurans from Salicylamide Derivatives and its Application toward the Total Synthesis of Anigopressin A, SISOIUM 2005, Montreal, Quebec, April 28-29, 2005.

134. Alessi, M., Reed, M., Snieckus, V. Studies Towards the Total Synthesis of Isoprekinamycin. An Approach Through a New O $\rightarrow$ C 1,5 Vinyl Carbamoyl Translocation. SISOIUM 2005, Montreal, Quebec, April 28-29, 2005.

135. Ogilvie, K., Snieckus, V. The DoM - Halogen Dance Combo, SISOIUM 2005, Montreal, Quebec, April 28-29, 2005.

136. Lai, P., Lopez, S., Alessi, M., Green, L., Ogilvie, K., Snieckus, V. In situ Directed orthoMetalation - Boronation Suzuki-Miyaura Cross Coupling of Pyridine Derivatives, SISOIUM 2005, Montreal, Quebec, April 28-29, 2005.

137. Macklin, T., Snieckus, V. The N,N-diethylsulfamate as a New Directed ortho Metalation Group and Transition Metal Catalyzed Cross Coupling Partner, SISOIUM 2005, Montreal, Quebec, April 28-29, 2005

138. Snieckus, V. Boron as a Guide to Synthetic Methods linked to Aromatic Metalation, Pacifichem 2005, Honolulu, Hawaii, December 15-21, 2005.

139. Snieckus, V. C-H Bond activation by directed ortho Metalation, Pacifichem 2005, Honolulu, Hawaii, December 15-21, 2005.

140. Snieckus, V. Directed ortho Metalation - based Strategies for Condensed Aromatics and Heteroaromatics, DIOXIN 2005, Toronto, Ontario, August 25, 2005.

141. Blackburn, T., Alessi, M. Snieckus, V. Suzuki Cross coupling with in situ Dealkylation. A New Synthesis of Benzofuran. SOUCSS, Toronto, Ontario, March 18, 2006.

142. Alessi, M., Snieckus, V., Reed, M.A., New aromatic anionic synthetic methods. Towards the total synthesis of schumanniophytine. 12th Symposium on the Latest Trends in Organic Synthesis, St. Catharines, ON, August 9-12, 2006.

143. Macklin, T. New Aromatic Anionic Synthetic Methods. Towards the Total Synthesis of Schumanniophytine. ACS San Francisco, August 10 - 14, 2006.

144. Demchuk, O.M.; Snieckus, V. A Mixed Naphthyl-Phenyl Phosphine Ligand Motif for Suzuki, Heck, and Hydrodehalogenation Reactions. Balticum Organicum Syntheticum, Tallinn, Estonia, June 25-29, 2006.

145. Macklin, T. New Aromatic Anionic Synthetic Methods. Towards the Total Synthesis of Schumanniophytine. ACS Chicago, March 25-29, 2007.

146. J. Morey, T. Rantanen, V. Snieckus. Towards the Total Synthesis of Furostifoline and its Analogues. SOUCSS, Kingston, Ontario, March 15, 2008.

147. Markewicz, T., Snieckus, V., Gan, W., Lemieux, R. Synthesis of New Phenanthrenedione Liquid Crystals Via a Combined Directed Metalation Cross-Coupling Route. SOUCSS, Kingston, Ontario, March 15, 2008.

148. Nguyen, T., Alessi, M., Reed, M., Patel, J., Zhao, Y., Snieckus, V. Studies Towards the Total Synthesis of Isoprekinamycin. SOUCSS, Kingston, Ontario, March 15, 2008.

149. Groom, K., Snieckus, V. Toward the preparation of Chromopyrrolic ACid (CPA) as a probe of RebP enzyme structure and function, American Chemical Society, New Orleans, Louisiana, April 6-10, 2008.

150. Rantanen, T., Snieckus, V., Ni-catalyzed borylation of haloaromatics and -heteroaromatics, 
American Chemical Society, New Orleans, Louisiana, April 6-10, 2008.

151. Rantanen, T., Snieckus, V., Flatland Metalation. Aiming for New Synthetic Methodologies for Aromatics and Heteroaromatics, American Chemical Society, NERM, Burlington, Vermont, June 29-July 2, 2008.

152. Board, J., Wang, J. McCubbin, A., Jin, M., Crew, A., Laufer, R., Mulvihill, M., Snieckus, V. Palladium Catalyzed Direct Heck Coupling at C-5 of Imidazo[1,5-a]Pyrazines, American Chemical Society, NERM, Burlington, Vermont, June 29-July 2, 2008.

153. Wagner, H,, Board, J., Snieckus, V., Advances In Directed Ortho Metalation Chemistry. towards the Development of a Boron Based Directing Metal Group, American Chemical Society, NERM, Burlington, Vermont, June 29-July 2, 2008.

154. Ascic, E., Harmata, M., Månsson, K., Wärnmark, K., Snieckus, V., Towards Synthesis of New Tröger's Base Derivatives, American Chemical Society, NERM, Burlington, Vermont, June 29July 2, 2008.

155. Snieckus, V., Bonding Boron to Aromatic Metalation Synthetic Strategies, Matteson Symposium, Pullman, Washington, September 13, 2008.

156. Kåre B. Jørgensen, Toni Rantanen, Thilo Dörfler, and Victor Snieckus.Synthesis of 1Methylphenanthrene Metabolites by Directed Metalation Strategies. Canadian Society for Chemistry 2009, Hamilton, Ontario May 30-June 3, 2009.

157. Jignesh J. Patel, Manlio Alessi and Victor Snieckus. Studies towards the Total Synthesis of Isoprekinamycin. Canadian Society for Chemistry 2009, Hamilton, Ontario May 30-June 3, 2009.

158. Emilie David, Josefine Karlsson, Erhad Ascic, Victor Snieckus, Kenneth Wärnmark, Michael Harmata Synthesis of new Tröger's base derivatives. Canadian Society for Chemistry 2009, Hamilton, Ontario May 30-June 3, 2009.

159. Katherine A. Groom, V. Snieckus, D. Zechel. Investigating RebC-Mediated Chemoselective Oxidation during Rebeccamycin Biosynthesis. Canadian Society for Chemistry 2009, Hamilton, Ontario May 30-June 3, 2009.

160. Gan, Wei; Snieckus, Victor; Lemieux, Robert. Synthesis of A New LC Material with Phenanthrene Core by A Combined Metalation-Cross Coupling Route. Canadian Society for Chemistry 2009, Hamilton, Ontario May 30-June 3, 2009.

161. Yigang Zhao, Victor Snieckus. Reduction of Tertiary Amides to Aldehydes by in situ Generation of Schwartz Reagent $\left[\mathrm{Cp}_{2} \mathrm{Zr}(\mathrm{H}) \mathrm{Cl}\right]$. Canadian Society for Chemistry 2009, Hamilton, Ontario May 30-June 3, 2009.

162. Hurst, T., Macklin, T., Hartmann, E., Kugel, W., Parisienne-La Salle, J.-C., Becker, M., Schneider, $\underline{\underline{C} .,}$ Marder, T., Snieckus, V., Synthesis of regioisomeric aryl boronates via complementary methodologies, International Conference on Heterocyclic Chemistry, St. John's, Newfoundland, August 2-8, 2009.

163. Yigang Zhao, Justin Morin, and Victor Snieckus, Reduction of tertiary amides to aldehydes by in situ generated Schwartz reagent $\left[\mathrm{Cp}_{2} \mathrm{Zr}(\mathrm{H}) \mathrm{Cl}\right]$. Link to the directed ortho metalation (DoM) reaction, International Conference on Heterocyclic Chemistry, St. John's, Newfoundland, August 2-8, 2009.

164. Yigang Zhao, Andrew Larkin, Victor Snieckus, Towards the synthesis of lysergic acid via a directed ortho metalation (DoM) strategy, International Conference on Heterocyclic Chemistry, St. John's, Newfoundland, August 2-8, 2009.

165. Wei Gan, Robert Lemieux, Victor Snieckus, Synthesis of A New Liquid Crystal (LC) Material with Phenanthrene Core by A Combined Metalation-Cross Coupling Strategy, International Conference on Heterocyclic Chemistry, St. John's, Newfoundland, August 2-8, 2009.

166. Josefine Karlsson, Erhad Ascic, Victor Snieckus, Kenneth Wärnmark, Michael Harmata, Synthesis of new Tröger's Base Derivatives, International Conference on Heterocyclic Chemistry, St. John's, Newfoundland, August 2-8, 2009.

167. Antoft-Finch, A., Blackburn, T., Snieckus, V. N,N-Diethyl O-Carbamate: Directed Metalation Group and Orthogonal Cross-Coupling Partner, $38^{\text {th }}$ SOUSCC, Toronto, ON, March 2010. 
168. Rantanen, T., Miller, R., Snieckus, V., So you think you can dance, pyridine? $239^{\text {th }}$ ACS Conference, San Francisco, CA, March 21-25, 2010.

169. C. Schneider, E. Broda, and V. Snieckus. Synthesis of aryl/heteroaryl sulfonamides boropinacolates and their application. 239 ${ }^{\text {th }}$ ACS Conference, San Francisco, CA, March 21-25, 2010.

170. E. David, C. Schneider, V. Snieckus, Investigating the chemoselectivity of RebC-assisted biosynthesis of rebeccamycin Directed ortho metalation approach to C-4 substituted 7-azaindoles, $239^{\text {th }}$ ACS Conference, San Francisco, CA, March 21-25, 2010.

171. Yigang Zhao, Victor Snieckus. Reduction of Tertiary Amides to Aldehydes by in situ Generation of Schwartz Reagent $\left[\mathrm{Cp}_{2} \mathrm{Zr}(\mathrm{H}) \mathrm{Cl}\right]$., Balticum Organicum Syntheticum 2010, Riga, Latvia, June 27-30, 2010.

172. da Silva, A., Snieckus, V., Regioselective Arylation of Fluorenone Skeleton via Combined Ruand Pd-Catalyzed Suzuki Cross Coupling Reactions, $93^{\text {rd }}$ CSC, Toronto, ON, May 30-June 3, 2010 .

173. Ricarda E. Miller, Victor Snieckus ${ }^{2}$ and Ulrich Groth, Unexpected One-Pot Sequential Halogen Dance - Anionic Ortho Fries Rearrangement Of $O$-Aryl Carbamates, Balticum Organicum Syntheticum 2010, Riga, Latvia, June 27-30, 2010.

174. Yigang Zhao, Justing Morin, Victor Snieckus, Reduction of Tertiary Amides to Aldehydes by in situ Generation of Schwartz Reagent $\left[\mathrm{Cp}_{2} \mathrm{Zr}(\mathrm{H}) \mathrm{Cl}\right]$. Link to the Directed ortho Metalation (DoM) Reaction. 10th Winter Conference on Medicinal and Bioorganic Chemistry, Steamboat Springs, Colorado, Jan 23-27, 2011.

175. Adam Friedman, Matthew Kitching, Yigang Zhao, Victor Snieckus, Directed ortho Metalation Route Towards C-7 Functionalized Benzimidazoles. 94 ${ }^{\text {th }}$ CSC, Montreal, QC, June 5-9, 2011.

176. Jenn Cosman, Victor Snieckus, Directed ortho Metalation Chemistry. Towards the Development of a Boron Based Directing Metal Group, 94 ${ }^{\text {th }}$ CSC, Montreal, QC, June 5-9, 2011.

177. Yigang Zhao, Justin Morin, Victor Snieckus, Mild Selective Reduction of Tertiary Amides to Aldehydes via the in situ Generated Schwartz Reagent $\left[\mathrm{Cp}_{2} \mathrm{Zr}(\mathrm{H}) \mathrm{Cl}\right]$. Link to the Directed ortho Metalation (DoM) Reaction, $94^{\text {th }}$ CSC, Montreal, QC, June 5-9, 2011.

178. Tim Hurst, Alcides da Silva, Victor Snieckus, Negishi Cross Coupling of Vinylzincs with Aryl Halides and Triflates. A Friedel-Crafts Equivalent for the Regioselective Synthesis of Polysubstituted Acetophenone Derivatives, $94^{\text {th }}$ CSC, Montreal, QC, June 5-9, 2011.

179. Cedric Schneider, Alcides da Silva, Victor Snieckus, Chemoselective Catalytic C-O Activation. Construction of Polyarylated Fluorenones via Ru- and Pd-Catalyzed Suzuki Cross-Coupling Reactions, $94^{\text {th }}$ CSC, Montreal, QC, June 5-9, 2011.

180. Ricarda Miller, H. Fan, Ulrich Groth, Victor Snieckus, Synthesis of Schumanniophytinderivatives via key Remote Anionic Fries rearrangement, $23^{\text {rd }}$ ICHC, Glasgow, Scotland, July 31-August 4, 2011

181. Timothy E. Hurst, Matthew Kitching, and Victor Snieckus, Copper Catalyzed Cascade Interrupted by an Opportunistic Smiles Rearrangement: An Efficient Domino Approach to Dibenzoxazepinones, $4^{\text {th }}$ Annual Queen's Chemistry Graduate Symposium, Kingston, ON, September $7^{\text {th }}, 2011$.

182. Matthew Kitching, Adam Friedman, Yigang Zhao, Victor Snieckus, Towards the Regioselective Functionalization of C-7 Substituted Benzimidazoles, $4^{\text {th }}$ Annual Queen's Chemistry Graduate Symposium, Kingston, ON, September $7^{\text {th }}, 2011$.

183. Toni Rantanen, Jennifer Cosman, Victor Snieckus, Snieckus Innovations - Solving Synthetic Challenges and Learning Along the Way, $4^{\text {th }}$ Annual Queen's Chemistry Graduate Symposium, Kingston, ON, September $7^{\text {th }}, 2011$.

184. T.E. Hurst, M. O. Kitching, F. Lee and V. Snieckus, Copper-Catalyzed Cross-Coupling Interrupted by an Opportunistic Smiles Rearrangement: An Efficient Domino Approach to Dibenzoxazepinones, The $15^{\text {th }}$ Symposium on the latest trends in Organic Synthesis, St. Catharines, ON, August 8-12, 2012. 
185. M. Dalziel, M. Kitching, C. Schneider, A. Bailey, C. Crudden, V. Snieckus*, Dancing DMGs: Regioselective Functionalization of Azine or Azole in Azaindole, The 15th Symposium on the latest trends in Organic Synthesis, St. Catharines, ON, August 8-12, 2012.

186. Jennifer L. Cosman, Johnathan Board, Toni Rantanen, Victor Snieckus*, Serendipitous Discovery of an Efficient Synthesis of 4-Chloro-2H-Chromenes and Their Derivatives, The 15th Symposium on the latest trends in Organic Synthesis, St. Catharines, ON, August 8-12, 2012.

187. Suneel P. Singh, Adam Friedman, Matthew O. Kitching, Victor Snieckus, Selective metallation of substituted benzimidazole, The 15th Symposium on the latest trends in Organic Synthesis, St. Catharines, ON, August 8-12, 2012.

188. Jennifer L. Cosman, Johnathan Board, Toni Rantanen, Victor Snieckus, Serendipitous Discovery of an Efficient Synthesis of 4-Chloro-2H-Chromenes and Their Derivatives, 5th Annual Queen's Chemistry Graduate Symposium, Kingston ON, September 7, 2012.

189. Timothy Hurst, Synthetic Efforts Towards a New Treatment for Tuberculosis, 5th Annual Queen's Chemistry Graduate Symposium, Kingston ON, September 7, 2012.

190. Michael Dalziel, Dancing Directed Metalation Groups: Regioselective Functionalization of Azaindoles, 5th Annual Queen's Chemistry Graduate Symposium, Kingston ON, September 7, 2012.

191. Matthew Kitching, Towards the Regioselective Functionalization of C-7 Substituted Benzimidazoles, 5th Annual Queen's Chemistry Graduate Symposium, Kingston ON, September 7, 2012.

192. C.-H. F. Lee, K. G. Guimarães, T. E. Hurst, M. O. Kitching, A. J. M. da Silva, V. Snieckus, Towards the Total Synthesis of ( \pm )-Paralycolin A, SOUSCC 2013, McMaster University, Hamilton, ON, March 30 2013.

193. Christopher A. Ziebenhaus, Matthew O. Kitching, Jignesh Patel, and Victor Snieckus, Towards the Total Synthesis of Isoprekinamycin and the Related Fluostatin Natural Products, SOUSCC 2013, McMaster University, Hamilton, ON, March 30 ${ }^{\text {th }}, 2013$.

194. Victor Snieckus, It's Organic! The Imprint of David Harpp, 96th Canadian Chemistry Conference and Exhibition in Québec, Quebec, May 26-30, 2013.

195. Keller Guimarães, Victor Snieckus, Towards the Total Synthesis of (!CB1)-Paralycolin A, 96th Canadian Chemistry Conference and Exhibition in Québec, Quebec, May 26-30, 2013.

196. Michael Dalziel, Victor Snieckus, Dancing Directed Metalation Groups: Regioselective Functionalization of Azaindoles, 96th Canadian Chemistry Conference and Exhibition in Québec, Quebec, May 26-30, 2013.

197. Suneel Singh, Victor Snieckus, A Directed ortho-Metalation (DoM) Strategy for the Synthesis of C-7 (C-4) Substituted Benzimidazoles, 96th Canadian Chemistry Conference and Exhibition in Québec, Quebec, May 26-30, 2013.

198. Christopher A. Ziebenhaus, Matthew O. Kitching, Jignesh Patel, and Victor Snieckus, Towards the Total Synthesis of Isoprekinamycin and the Related Fluostatin Natural Products, 96th Canadian Chemistry Conference and Exhibition in Québec, Quebec, May 26-30, 2013.

199. Jenn Cosman, Victor Snieckus, Serendipitous Discovery of an Efficient Synthesis of 4-Chloro2H-Chromenes and Their Derivatives, 96th Canadian Chemistry Conference and Exhibition in Québec, Quebec, May 26-30, 2013.

\section{GRADUATE STUDENTS}

\section{(a) Ph.D. Theses}

1. G.Y.-P. Kan (1970): Selected Studies of N-Iminopyridinium Ylides and Pyrylium and Thiopyrylium Salts. External Examiner: P.A.S. Smith, University of Michigan, USA. 
2. K.S. Bhandari (1970): Catalytic, Photochemical and Thermal Decomposition of Indole-2diazoketone. Chemistry of 2-(2-Aminoethyl) Indole Derivatives. External Examiner: S. McLean, University of Toronto.

3. J.A. Eenkhoorn (1971): Approaches Towards the Synthesis of the Uleine-Type Alkaloids. External Examiner: E. Warnhoff, University of Western Ontario.

4. H.O. Bernhard (1971): Synthetic Approaches Towards the Rhoeadine Papaverrubine Alkaloids. Synthesis of Schoepf-Schweickert Amine VI. External Examiner: D.B. MacLean, McMaster University.

5. S.O. de Silva (1973): Wittig Synthesis and Photocyclization Reactions of some 2- and 3- (2-Aryl) Vinylindoles. External Examiner: E. Wenkert, Rice University, USA.

6. I. Ahmad (1976): Synthetic Routes to the Rhoeadine and the Phthalideisoquinoline Alkaloids. External Examiner: M. Shamma, Penn. State University, USA.

7. D.J. Harris (1976): Organic and Organometallic Chemistry of 1,2-Diazepines. External Examiner: J. A. Moore, University of Delaware, USA.

8. F.W. Widmer (1977): Purification and Partial Characterization of Tryptophan-5-Hydroxylase. External Examiner: N. Cohen, NIH, Bethesda, MD, USA.

9. G.B. Mpango (1980): Metalated a,b-Unsaturated Amides in Organic Synthesis. Application to the Synthesis of 1-Aryltetralin Lignans. External Examiner: P. Beak, University of Illinois, USA.

10. M. Majewski (1982): Amide Dienolate Alkylations. Synthesis of Pyrethroid Amides. External Examiner: M.W. Rathke, Michigan State University, USA.

11. R.J. Mills (1985): Silicon in Benzamide Directed Metalation Reactions. External Examiner: J.C. Martin, University of Illinois, USA.

12. J.N. Reed (1985): Ortho-Amination of Lithiated Tertiary Benzamides, Synthesis of Anthranilamides, Acridones and the Antibiotic Anthramycin. External Examiner: E. Vedejs, University of Wisconsin, USA.

13. M.A.J. Miah (1985): The Tertiary Amide and Carbamoyloxy Groups in Aromatic Directed Metalation. Synthesis of Polysubstituted Benzene and Pyridine Derivatives. External Examiner: R. Fraser, University of Ottawa.

14. M.I. Mumtaz (1986): Dilithiated Aliphatic Diamides. Methodology and Synthesis of Lignan Natural Products. External Examiner: A. Schultz, Rennsalaer Polytechnic Institute, USA.

15. J.R. Green (1987): I. y-Silylated a,b-Unsaturated Amides: Preparation and Condensation Chemistry. II. Stereoselectivity in Amide Dienolate Formation. External Examiner: R. Schlessinger, University of Rochester, USA.

16. E.G. Doadt (1988): The Directed Aromatic Metalation Reaction. Synthesis of Anthraquinones, Polysubstituted Thiophenes and, Via Benzynes, 1,4-Epoxynaphthalenes. External Examiner: G. Gribble, Dartmouth College, USA. 
17. M.A. Siddiqui (1990): The Directed ortho Metalation and Palladium Catalyzed Aryl-Aryl Cross Coupling Reactions. Synthesis of Heteroaromatics and Alkaloids. External Examiner: D. Comins, North Carolina State University, USA.

18. J.-m. Fu (1990): Combined Directed ortho Metalation - Pd(O) - Catalyzed Cross Coupling Strategies. Regiospecific Synthesis of 9-Phenanthrols and Fluorenones including Dengibsinin. External Examiner: W. Wulff, University of Chicago, USA.

19. X. Wang (1992): Synthetic Applications of Directed ortho Metalation and Palladium Catalyzed Aryl-Aryl Cross Coupling Reactions. External Examiner: L. Hegedus, Colorado State University, USA.

20. Bao-ping Zhao (1994): Remote Directed Metalation. A Regiospecific and Efficient Preparation of Fluorenone Derivatives Including Natural Products. External Examiner: L.S. Liebeskind, Emory University, USA.

21. F. Beaulieu (1994): The Thiocarbamate as a Dual Directed Metalation and Cross Coupling Group Intramolecular 1,5-Hydrogen Atom Transfer in Aromatic Amides. External Examiner: G. Stork, Columbia University, USA.

22. M. Tsukazaki (1995): Synthetic Application of O-Vinyl and O-Arylcarbamates. Synthesis of Dihydroxyethylene Dipeptide Isosters and Benzofuran 5-Lipoxygenase Inhibitor Analogues and Synthesis and Reactivities of 2-Substituted Allyltrimethylsilane and 3,4-Pyridyne Precursors. External Examiner: P. Parsons, University of Sussex, UK.

23. M. Campbell (1996): I. Mechanistic Investigations into the Suzuki Cross Coupling Reaction and Its Application to Synthesis. II. Synthesis of Macrocyclic Ligands for the Complexation and Detection of Aromatic Pollutants. External Examiner: S.L. Buchwald, Massachusetts Institute of Technology, USA.

24. C. Quesnelle (1996): Methodological and Comparative Studies on Directed ortho Metalation of Aryl tert-Butyl Sulfoxides and Ni(0)-Catalyzed Cross Coupling of Aryl Triflates and $O$-Aryl Carbamates. Studies Directed Toward the Total Synthesis of Sterigmatocystin. External Examiner: L.S. Hegedus, Colorado State University, USA.

25. Clint A. James (1998): The Directed Remote Metalation - Carbomyl Migration Reaction. Total Syntheses of the Defucogilvacarcins and Arnottin I and Synthesis of Heteroaryylfused Benzopyranones. External Examiner: David Hart, Ohio State University, USA.

26. Sylvie Chamoin (2000): Aryl-Aryl Stille and Suzuki-Miyaura Cross Coupling Reactions on Solid Support. External Examiner: Thomas Hoye, University of Minnesota, USA.

27. Brian Chapell (2000): Directed orth Metalation for Synthesis of Chromenes and Indoles. External Examiner: Scott Denmark, University of Ilinois, Urbana-Campaign, USA.

28. Costa Metallinos (2001): Planar Chiral Ferroccenyl Ligands. Synthesis and Modification. External Examiner: Henri Kagan, Universite Paris Sud, France.

29. Brian Chauder (2001): Combined Directed ortho Metalation-Transition Metals Catalyzed Coupling Reactions. External Examiner: Marc Snapper, Boston College, USA. 
30. Stephen MacNeil (2002): The Tertiary Sulfonamide as a Directed Metalation Group. External Examiner: Derrick Clive, University of Alberta.

31. Robert Milburn (2003): Tertiary Aryl Sulfonamides as New Cross Coupling Partner for Ni Catalyzed Kumada-Corriu-Tamao Reductive and C-C Bond Forming Cross Coupling Reactions. External Examiner: Andre Charette, Université de Montreal.

32. Adam McCubbin (2004). Directed Metalation Approach to Ferroelectric Liquid Crystals with Chiral Fluorenol Cores: The Effect of Restricted Rotation and Hydrogen Bonding on Polar Order. External Examiner: Danial Dyer, Southern Illinois University, USA.

33. Zhongdong Zhao (2006.): Direct ortho Metalation - Electrophile-Induced Ipso-Desilylation Reactions. Towards Unconventional Aromatic Substitution Patterns. External Examiner: Rob Batey, University of Toronto.

34. Todd Macklin (2007): The Development of Sulfamates as Latent Directed Metalation Groups. Total Synthesis of Schumanniophytine. Divergent Synthesis of Substituted Chromone 3and 8-Carboxamides External Examiner. M. Smith, III, Michigan State University, USA.

35. Manlio Alessi (2008): Towards the Synthesis of Isoprekinamycin. The phosphorodiamidate Directed Metalation Group. G. Molander, University of Pennsylvania, USA.

36. Wei Gan (2009): A combined metalation and cross-coupling route to a new smetic liquid crystal with a phenanthrene core. G. Bodwell, Memorial University, St. John's, Newfoundland.

37. Yigang Zhao (2010): Reduction of Tertiary Benzamides to Benzaldehydes by an in situGenerated Schwartz Reagent $(\mathrm{Cp} 2 \mathrm{Zr}(\mathrm{H}) \mathrm{Cl})$; Formal Synthesis of Lysergic Acid. Gunda George, University of Minnestoa.

38. Katherine Groom (2013): Investigation of the Chemoselectivity of RebC-Assisted Biosynthesis of Rebeccamycin and Synthesis of Stable Analogues of Chromopyrrolic Acid, Paul Harrison, McMaster University.

\section{(b) M.Sc. Theses}

1. E. Watson (1970): Selected Ketocarbene and Nitrene Reactions in Heterocyclic System.

2. A.H.E. Wu (1975): A formal Synthesis of the Complex Indole Alkaloids ( \pm )-Condyfoline and $( \pm)$ Tubifoline and $( \pm)$ Tubifolidine.

3. I.O.W. Tse (1976): Photochemical Preparation of Pyrrolo-[2,1-b] [3] benzazepines. A Synthetic Approach to Cephalotaxux Alkaloids.

4. R.I. Wojtyk (1977): The Synthesis of Tetrahydrocannabinol, its Tritiation by Isotopic Exchange, and the Synthesis of its Epoxides.

5. J.A. Oakleaf (1977): I. Alkylation of Metalated Unsaturated Amides. II. Computer Teaching of Organic Synthesis.

6. Mutus, B. (1977): Studies on Tryptophan-5-Monooxygenase. 
7. J.N. Reed (1979): I. Synthesis of the Isoindole [1,2-b] Isoquinoline and Isoindolo [1,2-b] [3] Benzazocene Ring Systems.

8. R. Billedeau (1983): Directed ortho Lithiation of Tertiary Benzamides. 1. Synthesis of Phthalide and Isocoumarin Natural Products. 2. $\mathrm{O} \rightarrow \mathrm{C}$ Silicon Migrations.

9. N. Altintas (1985): Synthetic Studies Towards Anthracyclinone Antibiotics.

10. M.J. Sharp (1986): Biaryls by the Cross-Coupling of Directed Metalation Derived Aryl Boronic Acids with Haloarenes.

11. C. Sloan (1987): Radical-Induced Cyclization Routes to Heterocycles.

12. K. Cullen (1988): Aza-Polycyclic Aromatic Hydrocarbons: A Review of the Literature.

13. H. Perrier (1989): Directed ortho Metalation of Phenyl and Pyridyl $t$-Butyl Sulfones and $t$-Butyl Sulfoxides.

14. C. Unrau (1991): The Design and Synthesis of Molecular Cavity Receptors for a-Amino Acids. Polyaryl Synthesis Using 2:1 Pd(O)-Catalyzed Boronic Acid Cross Coupling.

15. W. Wang (1991): Directed ortho and Remote Aromatic Metalation. Synthesis of Highly Substituted Biaryls, Dibenzo[b,d]Pyranones and 3,3'-Substituted-1,'-Bi-2-Naphthols.

16. J. Arora (1995): [1,5]-Hydrogen Atom Translocation in Thiobenzamides and Pyrimidinones. Mechanistic and Synthetic Studies.

17. A. Kinsman (1997): Directed ortho Metalation-cross Coupling Route to ortho-quinodimethanes. Synthesis of Benz[e]indoles.

18. K. Puumala (1997): I. Directed ortho Metalation and Cross Coupling Reactions of O- and S-Aryl Thiocarbamates. II. DoM and Cross Coupling Methodologies in Polysubstituted Naphthalene Synthesis

19. Stephen MacNeil (1997): Synthesis of Acridone Alkaloids by an Anionic Friedel Crafts Equivalent.

20. Brian A. Chauder (1998): Methodological Studies on the Phenylboronic Aid-mediated Synthesis of 2H-1-Benzopyrans. Total Synthesis of Plicadin, A Coumestan from Psoralea plicata.

21. Bing Miao (1998): Towards the Total synthesis of Karnamicin B2.

22. Robert Milburn (1999): Synthesis of 3,3'-Diarylsubstituted BINOLs.

23. Christopher Kendall (1999): The Directed ortho-metalation Suzuki-Miyaura Cross Coupling Connection towards the Total Synthesis of Dehydrorabelomycin.

24. Laine Green (2001): Synthesis of functionalized pyridine boronic acids and connections to Cross Coupling.

25. Chunfeng Yin (2001): Directed ortho Metalation (DoM) and Suzuki-Miyaura Cross Coupling. 
26. Claire Milburn (2002): The Directed ortho Metalation Reaction of Aryl O-Carbamates on Solid Support.

27. Andrew Larkin (2002): Studies Towards the Total Synthesis of ( \pm )-Lysergic Acid.

28. Roseanne Quinn (2002): Phosphorous-based Directed Metalation Groups: Suzuki-Miyaura Cross Coupling.

29. Brian Wilson (2004): Anionic ortho-Fries Rearrangement of N-Carbamoyl Diarylamines. Synthesis of the Acridone Alkaloid Junosidine.

30. Chris Jones (2005): Directed ortho Metalation - Cross Coupling Methodology of Naphthalene 1,8Bis-Diethylamide.

31. Kevin Ogilvie (2006): The DoM - Halogen Dance Combo: Synthesis of Tri- And TetraSubstituted Pyridines By Directed ortho Metalation - Halogen Dance Methodology.

32. Justin Morin (2006): The Peri-Metalation of Naphthalenes.

33. Ping-Shan Lai (2007): Directed ortho metalation-boronation Suzuki-Miyaura cross coupling leading to synthesis of azafluorenol core liquid crystals.

34. Thomas Blackburn (2009): Progress in directed ortho Metalation, and II.Generating chirality in periodic mesoporous organosilica.

35. Michael (in progress, co-supervisor: Andrew Evans): New Enantioselective Allylation Directed ortho Metalation Synthetic Methodology.

36. Jenn Cosman (in progress, co-supervisor: Andrew Evans): New Enantioselective Allylation Directed ortho Metalation Synthetic Methodology.

\section{CURRENT RESEARCH GROUP}

\section{GRADUATE STUDENTS}

Katie Groom

Michael Dalziel

Jenn Cosman
Ph.D. $\quad$ Joint (D. Zechel) 2005

M.Sc. Joint (A. Evans) 2013

M.Sc. Joint (A. Evans) 2013
Complete

Active

Active

\section{POST DOCTORAL FELLOWS AND VISITING SCIENTISTS}

Toni Rantenan Johnathan Board

Timothy Hurst Matthew Kitching

Ondrej Kysilka

Suneel Singh Ashish Maheta
Chemistry Manager, Snieckus Innovations

Chemistry Manager, Snieckus Innovations

Postdoctoral Fellow

Postdoctoral Fellow

Postdoctoral Fellow

Postdoctoral Fellow, Snieckus Innovations

Visiting Scientist, Snieckus Innovations 


\section{GRADUATE STUDENTS AND POST DOCTORAL FELLOWS, 1967-PRESENT}

\section{STUDENT (M.Sc.)}

1. Altintas, N.

2. Arora, J.

3. Balint, R.

4. Benesch, L

5. Blackburn, T.

6. Billedeau, R.

7. Miao, B.

8. Cheng, W.L.

9. Cullen, K.

10. Green, L.

11. Holder, M.

12. Ionica, I.

13. Jones, C.

14. Joseph, B.

15. Kendall, C.

16. Kinsman, A.

17. Larkin, A.

18. Laufer, R.

19. Miao, B.

20. Milburn, C.

21. Morin, J.

22. Mutus, B.

23. Oakleaf, J.

24. Oglivie, K.

25. Palencia, H. (CIDA Fellow)

26. Perrier, $\mathrm{H}$.

27. Puumala, K.

28. Quinn, R.

29. Reed, J.N.

30. Sharp, M.J.

31. Sloan, C.

32. Tse, I.O.W.

33. Unrau, C.

34. Wang, W.

35. Watson, E.

36. Wilson, B.

37. Wojtyk, R.I.

38. $\mathrm{Wu}, \mathrm{A}$.

39. Yin, Feng

\section{STUDENT (Ph.D.)}

1. Ahmad, I.

2. Alessi, M.

3. Alves, G.B.C.(CNPq Fellowship)

4. Anctil, E.

5. Beaulieu, F.

\section{PRESENT POSITION}

University of British Columbia

Research Chemist, NPS, Mississauga, ON

Head, Anal Lab, MTC, Waterloo

unknown

Research Chemist, 3M, London, ON

Research Chemist, Roche, Palo Alto,CA

CIDtech Research Inc. Cambridge, ON

unknown

Brandon, Manitoba

Medical School, Dalhousie, NS

Brock University

Research Chemist, Toronto, ON

M.Sc. 200?, Queen's U

Research Chemist, NPS, Mississauga, ON

AstraZeneca, Montreal, Que

Research Chemist, Apotex (BCI) Brandford, ON

Research Chemist, GSK, Raleigh, NC

Group Leader, OSI, NJ

Group Leader, CIDtech, Cambridge, ON

Chemist, Amgen, Thousand Oaks, CA

Cancer Research Institute, Toronto, ON

Professor, University of Windsor, ON

Brampton, ON

M.Sc. Student, 2003-, Queen's U

Ph.D. Student, U. Nebraska

Chemist, Amgen, Thousand Oaks, CA

Chemist, Research Triangle Park, NC

Research Chemist, GSK, Raleigh, NC

Professor, Thompson Rivers University, BC

Head, Process Labs, GSK, Raleigh, N.C.

Research Chemist, BMS, CT

University of Western Ontario, ON

Teacher's College, U Toronto, ON

Research Chemist, BMS, CT

Birmingham, England

Chemist, GSK, Raleigh, NC

Surgeon, Ottawa, ON

Connaught Laboratories, Toronto, ON

Chemist, Pfizer-Agouron, San Diego, CA

\section{PRESENT POSITION}

Ministry of the Environment, Toronto, ON

Vanier College, Quebec

Chemist, U Rio de Janeiro, Brazil

Teacher, Montreal, Quebec

Research Chemist, BMS, Candiac, Que 
6. Bernhard, H.O.

7. Bhandari, K.S.

8. Brustle, S. (GDCh Fellowship)

9. Cai, X.

10. Campbell, $\mathrm{M}$.

11. Chapell, B.

12. Chauder, B

13. Chamoin, $\mathrm{S}$.

14. Da Silva, A. (CNPq Fellowship)

15. De Silva, S.O.

16. Doadt, E.G.

17. Eenkhoorn, J.A.

18. Espuga, P.(Spain Fellowship)

19. Felding, J. (Visiting Ph,D. Student)

20. Fu, J.M.

21. Focken, T. (GDCh Fellowship)

22. Garcia, E. (Spain Fellow)

23. Green, J.R.

24. Harris, D.J.

25. Hundsdorf, T.(German Fellowship)

26. James, C.

27. Kan, G. Y. P.

28. Kauch, M. (Humboldt Fellowship)

29. Koning, B.J. (STIR Fellow)

30. Macklin, T.

31. MacNeil, S.

32. Maillet, M. (France Govt Fellowship)

33. Majewski, M.

34. McCubbin, A.

35. Metallinos, $\mathrm{C}$.

36. Miah, M.A.J.

37. Milburn, R.

38. Mpango, G.B.

39. Miao,G.

40. Mills, R.J.

41. Mumtaz, M.I.

42. Pla, A. (Spain Fellowship)

43. Quesnelle, C.

44. Reed, J.N.

45. Rodrigues, D. (CNPq Fellowship)

46. Schneurch, M. (Austrian Fellowship)

47. Siddiqui, M.A.

48. Stefinovic, $M$.

49. Tsukazaki, M.

50. Wang, X.

51. Widmer, F.W.

52. Zhao, B.P.

53. Zhao, Y.

54. Zhao, Z.
Director, Seigfried AG, Switzerland

Teacher, Strathcona Lodge School, BC

Ph.D. Student, U Erlangen-Nurnberg

Ph.D. Student, 200?- Queen's U

Computational Chemist, Raleigh, NC

Professor, Mohawk College, ON

Research Chemist, GSK, Raleigh, NC

Research Chemist, Novartis, Suisse

Professor, U Rio de Janeiro, Brazil

Instructor, University of Waterloo, ON

Principal, Bluevale, Kitchener, ON

Ordained Minister, somewhere

Ph.D. Student, U Barcelona, Spain

Res Assoc, U Copenhagen, Denmark

Director, Xenon Gen, Burnaby, BC

PDF, U. Montreal (A. Charette)

Ph.D. Student, U de Pais Vasco, Spain

Professor, U of Windsor, ON

Head,Govt Anal Labs, Perth, Australia

Ph.D. Student, U Darmstadt, Germany

Research Chemist, BMS, Montreal, Que

Head, Anal Labs, Environ Canada, BC

Ph.D. received, U Munster, Gemany

Ph.D. received, U Gronigen, Netherlands

Research Chemist, Takeda Pharmaceuticals, CA

Professor, Wilfred Laurier U, ON

Chemist, Rhone-Poulenc, France

Professor, U of Saskatchewan

Professor, U. Winnipeg, Manitoba

Professor, Brock University, ON

Professor, U of Rajshahi, Bangladesh

Senior Research Chemist, Amgen, CA

Professor, Makerere University, Uganda

Research Chemist, Arquel, Boston, MA

Head, Process, SKF, Philadelphia, PA

Chemist, Dow Chemical Co. Sarnia, ON

Ph.D. Student, U de Girona, Spain

Group Leader, BMS, Candiac, Que

Professor, Thompson Rivers University, BC

Ph.D. Student, U Rio de Janeiro, Spain

Ph.D. Student, Tech U. Wien, Austria

Director, Shering-Plough, Boston, MA

Research Chemist, Sanofi, Austria

Group Leader, Roche Japan

Research Chemist, Amgen, CA

Director, Biotech. Inc. Denmark

Research Chemist

Postdoctoral Fellow, Snieckus Innovations

Research Chemist, Gilead Inc. 


\section{POST DOCTORAL FELLOWS AND VISITING SCIENTISTS}

\section{PRESENT POSITION}

1. Alo, B.(NSERC/CIDA Fellow)

2. Alves, T. (CNPq Fellowship)

3. Aubert, T. (NATO Fellow)

4. Bientinesi, I.

5. Blanchet, J.

6. Bower, J.

7. Brandao, M. (CAPES Fellowship)

8. Ellen Broda (Visiting M.Sc.)

9. Brough, P.

10. Bury, P.

11. Chattopadhyay S.(Visiting Professor)

12. Chieffi, A. (CNPq Fellowship)

13. Chong, R.

14. Coehlo, A. (CNPq Fellowship)

15. Cox, P.

16. Cuevas, C. (NATO Fellowship)

17. Da Silva, Alcides

18. David, Emilie

19. De Silva, S.O.

20. Derdau, V. (DAAD Fellowship)

21. Dexter, C. (Royal Soc Fellowship)

22. Dmitirienko, G.I.

23. Egestad. B. (Swedish Fellowship)

24. Emslie, N. (Visiting Professor)

25. Engqvist, R.

26. Epsztajn, J. (Visiting Professor)

27. Faessler, J. (Suisse Fellowship)

28. Familoni, W. (CIDA/NSERC Fellow)

29. Fecher, A. (DAAD Fellowship)

30. Familoni, W. (Visiting Professor)

31. Gan, Wei

32. Garcia, J. (Spain Fellowship)

33. Gharpure,M.

34. Glanser, J.

35. Gray, M.

36. Greff. Z. (CIDA Fellowship)

37. Griffen, E.

38. Guillaneux, D. (France Fellowship)

39. Hartung, C. (Humboldt Fellow)

40. Henaff, N.

41. Houldsworth, S.

42. Shakil Hussain (Visitin Ph.D)

43. Iihama, T. (Visiting Scientist)

44. Iwao, $M$.

45. Iwema-Bakker, W. (Dutch Govt Fellow) 46. Jablonski, C.R.

47. Jagusch, T.
Professor, U Lagos, Nigeria

U Minas Gerais, Brazil

Chemist, Rhone-Poulenc, France

U Pisa, Italy

Chemist, IRCOF Rouen, France

Research Chemist, Robotech, UK

Professor, U Minas Gerais, Brazil

Ludwig-Maximillians University, Germany

Research Chemist, Ribotargets, UK

Gr Leader, Manchester, UK

Professor, Jadavpur U, India

Professor, U Rio de Janeiro, Brazil

Chemist, U of Waterloo, ON

Chemist, U Rio de Janeiro, Brazil

Director of Research, Sanofi-Aventis, NJ

Research Chemist, GSK, Spain

Professor, Brazil

Postdoctoral Fellow, Université Rouen, France

Instructor, $\mathrm{U}$ of Waterloo, $\mathrm{ON}$

Research Chemist, Aventis, Germany

Gr Leader, Rhodia Pharma, UK

Professor, U of Waterloo, ON

Gr Leader, Neurosearch, Denmark

Professor, U of Natal, South Africa

Chemist, Astra-Zeneca, Sweden

Professor, Lodz University, Poland

Chemist, Zurich, Switzerland

Professor, U Lagos, Nigeria

Chemist, Pharma, Germany

University of Nigeria, Lagos, Nigeria

Postdoctoral Fellow, U. Ottawa

Professor, U de Navarra, Spain

PDF, VPI, Blacksburg, VA

unknown

Gr Leader, GSK, UK

Chemist, EGIS, Budapest, Hungary

Gr Leader, AstraZeneca, UK

Research Chemist, France

Research Chemist, Axxima, Germany

Chemist, Rhone Poulenc, France

Director, Dalton Chem, Toronto, ON

University of Karachi, Pakistan

Research Chemist, Nippon Soda, Japan

Professor, Nagasaki University, Japan

Chemist, Solvay, The Netherlands

Professor, Memorial U, Nfld

MercaChem, The Netherlands 
48. Jin, Z.

49. Johnson, M.

50. Jung, $M$.

51. Josephine Karlesson (Visiting, M.Sc.)

52. Kandil, A.

53. Kalinin, A. (NATO Fellowship)

54. Katsuura, K.

55. Kimachi, T.

56. Korolev, D.

57. Kothadaraman, S.

58. Lane, C.

59. Leite, M.(CNPq Fellowship)

60. Lin, M. S.

61. Lopez, S. (Visiting Professor)

62. Mahalanabis, K.K. (CIDA Fellow)

63. Mallet, M. (Govt France Fellowship)

64. McKibben, B.

65. Mercia, S.(CNPq Fellowship)

66. Miao, G.

67. Mohri, S.-i. (Visiting Scientist)

68. Mohri, K. (Visiting Professor)

69. Moriya, $\mathrm{T}$.

70. Moreau, P. (Govt France Fellowship)

71. Mizufune, H. (Visiting Scientist)

72. Nerdinger, S. (DAAD Fellowship)

73. Christian Nilewski (Visiting, M.Sc.)

74. Nolte, B. (DAAD Fellowship)

75. Parsons, A. (South Africa Fellowship)

76. Patel, J.

77. Patil, P.

78. Prien, O.

79. Rajapaksa, D.

80. Mark Reed

81. Riebel, P.

82. Roe, D.

83. Rogers-Evans, $\mathrm{M}$.

84. Roglans, A. (Spain Fellowship)

85. Scharl, Heiko

86. Schneider, Cedric

87. Schwaller, J.C. (NATO Fellow)

88. Sengupta, S.

89. Sibi, M.

90. Somera, N. (CAPES Fellowship)

91. Sotomayor, N. (Spanish Fellowship)

92. Superchi, S. (Italian Fellowship)

93. Szillat, H (Humboldt Fellowship)

94. Thomas, M.T.

95. Tinkl, $\mathrm{M}$.

96. Troisi, L. (Visiting Professor)
Chemist, Digital Chem, Toronto, ON

Research Chemist, AMRI, Albany, NY

Research Chemist, BASF

Lund University, Sweden

Chemist, Connaught Labs, Toronto, ON

Group Leader, Merck Sharp \& Dohme, Rahway, USA

Manager, Tosoh Corp, Japan

Professor, Kyoto U, Japan

Russia

Research Chemist, MSD, W Point, NJ

Research Chemist, Pfizer, UK

Chemist, U Minas Gerais, Brazil

Research Chemist Sloan Kettering, ILL

Professor, U S. Bolivar, Venezuela

Professor, Jadavpur University, India

Chemist, Rhone-Poulenc, France

Gr Leader, Boehringer-Ingelheim, NJ

Ph.D. student, U. Rio de Janeiro, Brazil

Research Chemist, ArQule, Boston, MA

Head, QC Labs, Kyowa Hakko, Japan

Professor, Showa College, Japan

Research Chemist, somewhere Japan

Res Associate, U d'Orleans, France

Research Chemist, Takeda Co, Japan

Research Chemist, Sanofi-Aventis, Austria

Post doctoral fellow, Scripps, CA

Research Chemist, Alantos Pharmaceuticals, Germany

Exec Dir, Chamber of Mines, S. Africa

GVK, India

Research Director, CIDtech, Cambridge, ON

Research Director, Schering AG, Germany

PDF, U of North Texas, TX

Group Leader, Treventis, Dalhousie, NS

Director Process Res, DSM, Germany

Research Director, Torcan Chemicals, ON

Group Leader, Roche, Basel, Switzerland

Professor, U Girona, Spain

Research Chemist, Austria

Postdoctoral Fellow, Université Rouen, France

Res Chemist, Rhone-Poulenc, France

Professor, Jadvapur University, India

Professor, U of North Dakota, ND

Professor, U Mato Grosso do Sul, Brazil

Professor, U del Pais Vasco, Spain

Professor, U Potenza, Italy

Research Chemist, Aventis, Germany

Chemist, Sunnybrook Med, Toronto, ON

Group Leader, CIBA, Basel, Suisse

Professor, U Lecce, Italy 
97. Tschamber, T (NATO Fellow)

98. Tsuchiya, T. (Visiting Professor)

99. Veith, U.

100. Watanabe, M.

101. Wicki, M.(Suisse Fellowship)

102. Wiesner, C.J.

103. Williams, $\mathrm{K}$.

104. Wong, P.L.

105. Venkateswarlu, R. (Visiting Professor)

106. Yin, C.

107. Zani, C. (CNPq Fellowship)

108. Zhou, Z.

109. Zumbansen, Kristina

110. Dr. Oleg Demchuk (NSERC/NATO)

111. Dr. Barbel Wittel

112. Dr. Till Vogel
Res Associate, U Haut Alsace, France Professor, Hokuriku U, Japan

Research Chemist, Lonza, Switzerland Professor, Nagasaki U (†14.03.00)

Group Leader, 3M Canada, London, ON Head, Res and Productivity Council, NB University of British Columbia Chemist, Scripps Institute, Philippines

Professor, Andhra U, India

Chemist, Toronto, ON

Group Leader, Pesquisas- FIOCRUZ, Brazil Professor, U Beijing, China

Visiting M.Sc Student, Universitat Münster

Professor, Maria Curie-Skłodowska University, Lublin, Poland

Archimica, Stuttgart, Germany

BASF, Germany 